\title{
Second order analysis for strong solutions in the optimal control of parabolic equations
}

\author{
Térence Bayen* $\quad$ Francisco J. Silva ${ }^{\dagger \ddagger}$
}

December 16, 2014

\begin{abstract}
In this paper we provide a second order analysis for strong solutions in the optimal control of parabolic equations. We consider the case of box constraints on the control and final integral constraints on the state. In contrast to sufficient conditions assuring quadratic growth in the weak sense, i.e. when the cost increases at least quadratically for admissible controls uniformly near to the nominal one (see e.g. $[16,26]$ ), our main result provides a sufficient condition for quadratic growth of the cost for admissible controls whose associated states are uniformly near to the state of the nominal one.

As a consequence of our results, for qualified problems with a strictly convex and quadratic Hamiltonian, we prove that both notions of quadratic growth coincide.
\end{abstract}

Keywords. Optimal control, semilinear parabolic equations, strong solutions, quadratic growth.

MSC. 49J20, 49K20, 35Q93.

\section{Introduction}

Besides its applications in several fields such as biochemistry [19], inverse problems [2] and biology [29], the optimal control of systems governed by nonlinear parabolic equations is one of the prototypes, together with the case of optimal control of hyperbolic systems, of optimization problems of evolutive systems. We refer the reader to the monographs $[23,28]$ for a rather complete review of the theory, the associated numerical analysis and some interesting applications (for the latter see in particular [23, Chapter 1]).

In this work we consider the optimal control problem of a semilinear parabolic equation where bounds constraints are imposed on the control and finitely many constraints are imposed on the state. Thus, our constraints are partially polyhedric in the sense of [11]. For the sake of clarity and also because of its analogy with the corresponding study for ordinary differential equations, we suppose that we have integral constraints on the final state. In the same spirit than [3], we can consider several notions of local solutions and of local solutions satisfying a quadratic growth property. Namely, we will say that $\bar{u}$ is a weak local solution of the problem if it minimizes the cost locally on the constraint set with respect to the $L^{\infty}$-norm. Moreover, if locally in the $L^{\infty}$ norm the difference between the cost of an admissible control $u$ and the cost of $\bar{u}$ is greater than a positive constant (independent

\footnotetext{
${ }^{*}$ Université Montpellier 2, Case courrier 051, 34095 Montpellier cedex 5, France (tbayen@math.univ-montp2.fr).

${ }^{\dagger}$ XLIM - DMI UMR CNRS 7252 Faculté des Sciences et Techniques, Université de Limoges (francisco.silva@unilim.fr).

${ }^{\ddagger}$ This research benefited from the support of the "FMJH Program Gaspard Monge in optimization and operation research", and from the support to this program from EDF.
} 
of $u$ ) times the square of the $L^{2}$ norm of $u-\bar{u}$, we will say that $\bar{u}$ is a weak local solution of the problem satisfying the quadratic growth condition in the weak sense. A characterization of the latter property, as well as its equivalence for some specific problems with a stronger notion of solution, are studied in [26] (see also $[17,5,16,10]$ and the references therein for other studies on the topic). Another type of local solution, whose definition goes back to the beginning of the theory of Calculus of Variations (see [22] for detailed analysis and [4] for a short survey), is the following: the nominal control $\bar{u}$ is a strong local solution of the problem if it minimizes the cost with respect to controls whose corresponding states are uniformly close to the nominal state. If the corresponding quadratic growth property holds true, then we will say that $\bar{u}$ is a strong local solution satisfying the quadratic growth property in the strong sense. We refer the reader to Section 2 for precise definitions of the notions explained above.

Our aim in this work is to study second order optimality conditions for strong solutions satisfying the quadratic growth condition. Following the ideas in [3], we first prove in Theorem 3.3 a decomposition result for the variation of the cost in terms of large and small perturbations in the $L^{\infty}$-norm of the nominal control. This decomposition is the basic tool to prove our main result, which is a sufficient condition for the quadratic growth property in the strong sense, proved in Theorem 4.4. The result states that if stronger forms of the classical Pontryagin's principle (see [18, 12, 14]) and of the usual coercivity of the second derivative of the Lagrangian, supposed to be a Legendre form, hold true, then the quadratic growth property in the strong sense is verified. In the case where no final constraints are considered, the Legendre form assumption can be dropped which yields a characterization of quadratic growth in strong sense (see Theorem 4.5). The latter result extends [3, Theorem 4.24], established for semilinear elliptic equations with pure control constraints.

As a consequence of the previous findings, we obtain in Section 5 some unexpected results. Under some continuity assumptions on the data, we have that if the Hamiltonian associated to the problem is quadratic and strictly convex with respect to the control and a constraint qualification condition holds true, then the notions of quadratic growth in the weak and strong sense are equivalent. When we consider only control constraints, the equivalence between the two notions of solutions holds true without any qualification condition (see Theorem 5.4). Of course, these arguments provide also the proof of the analogous statements in the case of optimal control problems of ordinary differential equations (see $[8,9]$ ) and of semilinear elliptic equations (see [3]).

The article is organized as follows: in Section 2 we recall some basic properties of semilinear parabolic equations, we state the optimal control problem and also our main assumptions. In Section 3 we study in detail a second order expansion of the Lagrangian of the problem and we prove the decomposition result. In sections 4 and 5 we prove our main results: the sufficient condition for quadratic growth in the strong sense, the characterization of this property in the case of pure control constraints and the equivalence with quadratic growth in the weak sense for strictly convex quadratic Hamiltonians. Finally, in the Appendix we provide the proof of some technical results stated in Section 3.

\section{Preliminaries}

From now on, we fix a non-empty bounded open set $\Omega \subseteq \mathbb{R}^{d}(d \in \mathbb{N})$ with a smooth boundary. Let us also fix $T>0$ and set $Q=\Omega \times] 0, T[$ and $\Sigma=\partial \Omega \times] 0, T$. For $s \in[1, \infty]$ and $k \in \mathbb{N}$, we denote by $\|\cdot\|_{s}$ and $\|\cdot\|_{W^{k, s}}$ the standard norms in $L^{s}(\Omega)$ and $W^{k, s}(\Omega)$, respectively. For $s_{1}, s_{2} \in[1, \infty]$, we set $L^{s_{1}, s_{2}}(Q):=L^{s_{1}}\left([0, T] ; L^{s_{2}}(\Omega)\right)$ (see e.g. [21]), which can be identified with the set of measurable 
functions $f: Q \rightarrow \mathbb{R}$ satisfying

$$
\|f\|_{s_{1}, s_{2}}:=\left(\int_{0}^{T}\left(\int_{\Omega}|f(t, x)|^{s_{2}} \mathrm{~d} x\right)^{\frac{s_{1}}{s_{2}}} \mathrm{~d} t\right)^{\frac{1}{s_{1}}}<\infty .
$$

Endowed with the norm $\|\cdot\|_{s_{1}, s_{2}}$ we have that $L^{s_{1}, s_{2}}(Q)$ is a Banach space. For $\left.s \in\right] 1, \infty[$ consider the space

$$
\mathcal{V}^{s}(Q):=L^{s}\left([0, T] ; W^{2, s}(\Omega) \cap W_{0}^{1, s}(\Omega)\right) \cap W^{1, s}\left([0, T] ; L^{s}(\Omega)\right),
$$

which endowed with the natural norm

$$
\|z\|_{\mathcal{V}^{s}}:=\|z\|_{s, s}+\left\|\partial_{t} z\right\|_{s, s}+\sum_{i=1}^{d}\left\|\partial_{x_{i}} z\right\|_{s, s}+\sum_{i, j=1}^{d}\left\|\partial_{x_{i} x_{j}} z\right\|_{s, s},
$$

is a Banach space. The following properties of the spaces $\mathcal{V}^{s}$ will play an important role in our results. For the proof we refer the reader to the monograph [6].

Lemma 2.1. Let $1<s \leq s_{i}<\infty$, with $i=1,2$. Then, the following assertions hold true:

(i) The space $\mathcal{V}^{s}(Q)$ is continuously embedded in $L^{s_{1}, s_{2}}(Q)$ if

$$
\frac{1}{s}-\frac{1}{s_{1}}+\frac{d}{2}\left(\frac{1}{s}-\frac{1}{s_{2}}\right) \leq 1 .
$$

Moreover, if (2.2) is a strict inequality, then the embedding is compact. In particular, if

$$
\left(1+\frac{d}{2}\right)\left(\frac{1}{s}-\frac{1}{s_{1}}\right) \leq 1
$$

then $\mathcal{V}^{s}(Q)$ is continuously embedded in $L^{s_{1}, s_{1}}(Q)$ (compactly embedded if the inequality is strict).

(ii) The space $\mathcal{V}^{s}(Q)$ is continuously embedded in $C^{0,1-1 / s}\left([0, T] ; L^{s}(\Omega)\right.$ ) (the space of $(1-1 / s)$-Hölder applications with values in $L^{s}(\Omega)$ ).

(iii) The space $\mathcal{V}^{s}(Q)$ is compactly embedded in $C(\bar{Q})$ (the space of continuous functions on $\bar{Q}$ ) if $(d+2) / 2 s<1$.

Given $\xi_{0} \in W_{0}^{2-\frac{2}{s}, s}(\Omega)$, with $s>(d+2) / 2, a \in L^{\infty, \infty}(Q)$ and $v \in L^{s, s}(Q)$ recall (see e.g. [20, Chapter 4, Theorem 9.1]) that the linear parabolic equation

$$
\begin{aligned}
\partial_{t} \xi-\Delta \xi+a(t, x) \xi & =v(t, x), \quad \text { in } Q \\
\xi & =0, \quad \text { in } \Sigma \\
\xi(0) & =\xi_{0} \quad \text { in } \Omega
\end{aligned}
$$

admits a unique strong solution $\xi\left[\xi_{0}, v\right] \in \mathcal{V}^{s}(Q)$, i.e. the equation is satisfied almost everywhere and $\xi\left[\xi_{0}, v\right](0, \cdot)$ (which is well defined by Lemma 2.1(i)) is equal to $\xi_{0}$. Moreover, there exists $c_{s}>0$ such that following estimate holds true (assuming $\xi_{0}=0$, for simplicity),

$$
\|\xi[0, v]\|_{\mathcal{V}^{s}} \leq c_{s}\|v\|_{s, s} .
$$

Using Aubin's Theorem (see [1,27]), it can be easily checked (see e.g. [26]) that the linear application $v \in L^{2,2}(Q) \rightarrow \xi[0, v] \in L^{2,2}(Q) \cap C\left([0, T] ; L^{2}(\Omega)\right)$ is continuous when $L^{2,2}(Q)$ and $L^{2,2}(Q) \cap$ $C\left([0, T] ; L^{2}(\Omega)\right)$ are endowed with the weak and the strong topologies, respectively. Finally, the following estimate (see [26]) will be also useful in Section 3

$$
\|\xi[0, v](\cdot, T)\|_{1}+\|\xi[0, v]\|_{1,1} \leq c_{1}\|v\|_{1,1}, \quad \text { for some } c_{1}>0 .
$$


Given $u \in L^{\infty, \infty}(Q)$ and $\varphi: Q \times \mathbb{R} \times \mathbb{R} \rightarrow \mathbb{R}$, we consider the following semilinear parabolic equation:

$$
\begin{aligned}
\partial_{t} y-\Delta y+\varphi(t, x, y, u) & =0 \quad \text { in } Q, \\
y(\cdot, \cdot) & =0 \quad \text { in } \Sigma, \\
y(0, \cdot) & =y_{0}(\cdot) \quad \text { in } \Omega .
\end{aligned}
$$

For a function $\psi=\psi(x, t, y, u)$ differentiable w.r.t. to the third and fourth coordinates we will write $\psi_{(y, u)}(t, x, y, u)$ for $D_{(y, u)} \psi(t, x, y, u)$ and $\psi_{y}(t, x, y, u), \psi_{u}(t, x, y, u)$ for the partial derivatives of $\psi$ w.r.t. $y$ and $u$ respectively. Similar notations will also be used for the second order derivatives. We assume that

(H1) (i) The initial state $y_{0}$ belongs to $W_{0}^{2-\frac{2}{s}, s}(\Omega)$, with $s>(d+2) / 2$.

(ii) The function $\varphi$ is measurable and for all $R>0$ there exists $c=c(R)>0$ such that

$$
-c\left(1+|y|^{2}\right) \leq \varphi(t, x, y, u) y \text { for all }(t, x, y) \in Q \times \mathbb{R},|u| \leq R .
$$

(iii) For a.a. $(t, x) \in Q$ the function $\varphi(t, x, \cdot, \cdot)$ is $C^{2}$, the application $(t, x) \rightarrow \varphi(t, x, \cdot, \cdot) \in C^{2}\left(\mathbb{R}^{2}\right)$ is measurable and there exists $c=c(R)$ such that $|(y, u)| \leq R$ and $\left|\left(y^{\prime}, u^{\prime}\right)\right| \leq R$ imply that

$$
\begin{gathered}
\left|\varphi_{(y, u)}(t, x, 0,0)\right|+\left|\varphi_{(y, u)^{2}}(t, x, 0,0)\right| \leq c \\
\left|\varphi_{(y, u)}(t, x, y, u)-\varphi_{(y, u)}\left(t, x, y^{\prime}, u^{\prime}\right)\right| \leq c\left(\left|y-y^{\prime}\right|+\left|u-u^{\prime}\right|\right), \\
\left|\varphi_{(y, u)^{2}}(t, x, y, u)-\varphi_{(y, u)^{2}}\left(t, x, y^{\prime}, u^{\prime}\right)\right| \leq c\left(\left|y-y^{\prime}\right|+\left|u-u^{\prime}\right|\right) .
\end{gathered}
$$

Denoting by $C^{\alpha, \beta}(\bar{Q})$ the space of functions defined on $\bar{Q}$ that are $\alpha$-Hölder continuous w.r.t. $t$ and $\beta$-Hölder continuous w.r.t. $x$, the following result holds true (see e.g. [12, 18, 26]).

Proposition 2.2. Under assumption (H1) for any $u \in L^{\infty, \infty}(Q)$, equation (2.7) admits a unique solution $y[u] \in \mathcal{V}^{s}(Q) \cap C^{\beta / 2, \beta}(\bar{Q})$ for all $\left.s \in\right] 1, \infty[$.

Remark 2.3. The result stated in the above Theorem holds true under weaker assumptions than (H1) (see $[12,18,26])$. As a matter of fact, the hypothesis on the second derivatives of $\varphi$ are not necessary for the well-posedness of (2.7), but they are fundamental for the second order expansions derived in the next section.

Let us consider $\ell: Q \times \mathbb{R} \times \mathbb{R} \rightarrow \mathbb{R}, \Phi: \Omega \times \mathbb{R} \rightarrow \mathbb{R}, \Phi_{E}: \Omega \times \mathbb{R} \rightarrow \mathbb{R}^{n_{E}}, \Phi_{I}: \Omega \times \mathbb{R} \rightarrow \mathbb{R}^{n_{I}}$ satisfying the following assumptions:

(H2) The function $\ell$ is measurable and satisfies (H1)(iii) with $\ell$ in place of $\varphi$. Moreover, if $\psi=$ $\Phi, \Phi^{i}, \Phi^{j}\left(i \in\left\{1, \ldots, n_{E}\right\}\right.$ and $\left.j \in\left\{1, \ldots, n_{I}\right\}\right)$ we have that

(i) $\psi$ is measurable.

(ii) For all $x \in \Omega, \psi(x, \cdot)$ is $C^{2}$, the application $x \mapsto \psi(x, \cdot) \in C^{2}(\mathbb{R})$ is measurable and there exists a constant $c=c(R)>0$ such that for all $y, y^{\prime} \in \mathbb{R}$ with $|y| \leq R,\left|y^{\prime}\right| \leq R$, we have that

$$
\begin{gathered}
\left|\psi_{y}(x, 0)\right|+\left|\psi_{y y}(x, 0)\right| \leq c, \quad\left|\psi_{y}(x, y)-\psi_{y}\left(x, y^{\prime}\right)\right| \leq c\left|y-y^{\prime}\right|, \\
\left|\psi_{y y}(x, y)-\psi_{y y}\left(x, y^{\prime}\right)\right| \leq c\left|y-y^{\prime}\right| .
\end{gathered}
$$

Let us define the functions $J: L^{\infty, \infty}(Q) \rightarrow \mathbb{R}, G_{E}: L^{\infty, \infty}(Q) \rightarrow \mathbb{R}^{n_{E}}, G_{I}: L^{\infty, \infty}(Q) \rightarrow \mathbb{R}^{n_{I}}$ and $G: L^{\infty, \infty}(Q) \rightarrow \mathbb{R}^{n_{E}+n_{I}}$ as

$$
\begin{aligned}
J(u) & :=\int_{Q} \ell(t, x, y[u](t, x), u(t, x)) \mathrm{d} t \mathrm{~d} x+\int_{\Omega} \Phi(x, y(T, x)) \mathrm{d} x, \\
G_{E}^{i}(u) & :=\int_{\Omega} \Phi_{E}^{i}(x, y[u](T, x)) \mathrm{d} x \quad \text { for } i=1, \ldots, n_{E}, \\
G_{I}^{j}(u) & :=\int_{\Omega} \Phi_{I}^{j}(x, y[u](T, x)) \mathrm{d} x \quad \text { for } j=1, \ldots, n_{I}, \\
G(u) & :=\left(G_{E}(u), G_{I}(u)\right) .
\end{aligned}
$$


Notice that, under assumptions (H1)-(H2), the functions $J, G_{E}$ and $G_{I}$ are well defined. Given $a, b \in L^{\infty, \infty}(Q)$ such that $a \leq b$ a.e. in $Q$, let us define the sets

$$
\begin{aligned}
\mathcal{K}_{1} & :=\left\{u \in L^{\infty, \infty}(Q) ; a(t, x) \leq u(t, x) \leq b(t, x) \text { a.e. in } Q\right\}, \\
\mathcal{K}_{2} & :=\left\{u \in L^{\infty, \infty}(Q) ; G_{E}^{i}(u)=0, G_{I}^{j}(u) \leq 0, i=1, \ldots, n_{E}, j=1, \ldots, n_{I}\right\}, \\
\mathcal{K} & :=\mathcal{K}_{1} \cap \mathcal{K}_{2} .
\end{aligned}
$$

We consider the optimal control problem

$$
\inf _{u \in L^{\infty, \infty}(Q)} J(u) \text { s.t. } u \in \mathcal{K} .
$$

When no final constraints are considered on the state, we will denote the problem by $\left(C P^{\prime}\right)$, i.e

$$
\inf _{u \in L^{\infty, \infty}(Q)} J(u) \text { s.t. } u \in \mathcal{K}_{1} .
$$

We consider the following notions of solution for problem $(C P)$ (the corresponding notions of solutions for $\left(C P^{\prime}\right)$ are obtained by replacing $\mathcal{K}$ by $\left.\mathcal{K}_{1}\right)$.

Definition 2.4. We say that

(i) $\bar{u}$ is a weak local solution of $(C P)$ if there exists $\varepsilon>0$ such that

$$
J(\bar{u}) \leq J(u) \text { for all } u \in \mathcal{K} \text { such that }\|u-\bar{u}\|_{\infty, \infty} \leq \varepsilon .
$$

If in addition, there exists $\alpha>0$ such that

$$
J(\bar{u})+\frac{\alpha}{2}\|u-\bar{u}\|^{2} \leq J(u) \text { for all } u \in \mathcal{K} \text { such that }\|u-\bar{u}\|_{\infty, \infty} \leq \varepsilon,
$$

we will say that $\bar{u}$ is a weak local solution of $(C P)$ satisfying the quadratic growth condition.

(ii) $\bar{u}$ is a $L^{s}$-weak local solution $(s \in[1, \infty[)$ of $(C P)$ if there exists $\varepsilon>0$ such that

$$
J(\bar{u}) \leq J(u) \text { for all } u \in \mathcal{K} \text { such that }\|u-\bar{u}\|_{s, s} \leq \varepsilon .
$$

If in addition, there exists $\alpha>0$ such that

$$
J(\bar{u})+\frac{\alpha}{2}\|u-\bar{u}\|^{2} \leq J(u) \text { for all } u \in \mathcal{K} \text { such that }\|u-\bar{u}\|_{s, s} \leq \varepsilon,
$$

we will say that $\bar{u}$ is a $L^{s}$-weak local solution of $(C P)$ satisfying the quadratic growth condition.

(iii) $\bar{u}$ is strong local solution of $(C P)$ if there exists $\varepsilon>0$ such that

$$
J(\bar{u}) \leq J(u) \text { for all } u \in \mathcal{K} \text { such that }\|y[u]-\bar{y}\|_{\infty, \infty} \leq \varepsilon,
$$

where $\bar{y}:=y[\bar{u}]$. If in addition, there exists $\alpha>0$ such that

$$
J(\bar{u})+\frac{\alpha}{2}\|u-\bar{u}\|^{2} \leq J(u) \text { for all } u \in \mathcal{K} \text { such that }\|y[u]-\bar{y}\|_{\infty, \infty} \leq \varepsilon,
$$

we will say that $\bar{u}$ is a strong local solution of $(C P)$ satisfying the quadratic growth condition.

Remark 2.5. Since for $u \in \mathcal{K}$ and $1 \leq s_{1} \leq s_{2}<\infty$ we have that

$$
\int_{Q}|u(t, x)|^{s_{2}} \mathrm{~d} t \mathrm{~d} x \leq M^{s_{2}-s_{1}} \int_{Q}|u(t, x)|^{s_{1}} \mathrm{~d} t \mathrm{~d} x,
$$

with $M:=\max \left\{\|a\|_{\infty, \infty},\|b\|_{\infty, \infty}\right\}$, it holds that $\|u\|_{s_{2}, s_{2}} \leq M^{1-\frac{s_{1}}{s_{2}}}\|u\|_{s_{1}, s_{1}}^{\frac{s_{1}}{s_{2}}}$ and thus the relative topologies of $L^{s_{1}, s_{1}}(Q) \cap \mathcal{K}$ and $L^{s_{2}, s_{2}}(Q) \cap \mathcal{K}$ are equivalent. This implies that for $s \in[1, \infty[$ the notions of $L^{s}$-weak solution and $L^{1}$-weak solution (respectively $L^{s}$-weak solution and $L^{1}$-weak solution satisfying the quadratic growth condition) are equivalent. As a consequence, using that Lemma 2.1(iii) and Lemma 3.1 in the next Section imply that $\|y[u]-\bar{y}\|_{\infty, \infty} \leq\|u-\bar{u}\|_{s, s}$ for s large enough, we obtain that $\|u-\bar{u}\|_{1,1} \rightarrow 0$, for $u \in \mathcal{K}$, implies that $\|y[u]-\bar{y}\|_{\infty, \infty} \rightarrow 0$. 


\section{Second order expansions and a decomposition result}

The Lagrangian $\mathcal{L}: L^{\infty, \infty}(Q) \times \mathbb{R}^{n_{E}} \times \mathbb{R}^{n_{I}} \rightarrow \mathbb{R}$ and the Hamiltonian $H: \bar{Q} \times \mathbb{R}^{3}$ associated to $(C P)$ are defined as

$$
\begin{aligned}
\mathcal{L}(u, \lambda) & :=J(u)+\lambda^{\top} G(u) \quad \text { where } \lambda=\left(\lambda_{E}, \lambda_{I}\right) \in \mathbb{R}^{n_{E}} \times \mathbb{R}^{n_{I}}, \\
H(t, x, y, p, u) & :=\ell(t, x, y, u)-p \varphi(t, x, y, u) .
\end{aligned}
$$

Now, let us fix $\bar{u} \in \mathcal{K}$ and set $\bar{y}:=y[\bar{u}]$. Given $\lambda=\left(\lambda_{E}, \lambda_{I}\right) \in \mathbb{R}^{n_{E}} \times \mathbb{R}^{n_{I}}$ define

$$
\Phi[\lambda](x, y):=\Phi(x, y)+\lambda_{E}^{\top} \Phi_{E}(x, y)+\lambda_{I}^{\top} \Phi_{I}(x, y) \text { for all } y \in \mathbb{R}, x \in \Omega .
$$

The adjoint state $\bar{p}_{\lambda}$ associated to $\bar{u}$ is defined as the unique weak solution of

$$
\begin{aligned}
-\partial_{t} p-\Delta p-H_{y}(t, x, \bar{y}, p, \bar{u}) & =0 \text { in } Q, \\
p & =0 \text { in } \Sigma, \\
p(T, \cdot) & =\Phi_{y}[\lambda](\cdot, \bar{y}(T, \cdot)) .
\end{aligned}
$$

By (H2)(ii) and the maximum principle one has $\bar{p}_{\lambda} \in L^{\infty, \infty}(Q)$.

The aim of this section is to provide a decomposition result for a second order expansion of the Lagrangian $\mathcal{L}$ in terms of the Hamiltonian and the adjoint state in the spirit of [3, Theorem 3.5]. The key issue is that we consider perturbations $v$ of $\bar{u}$ that are not necessarily small in the $\|\cdot\|_{\infty, \infty}$ norm. We will need first some precise informations about the effect of this type of perturbations on the application $u \rightarrow y[u]$. We collect in the following lemma some results of this type. For a function $\psi: Q \times \mathbb{R} \times \mathbb{R} \rightarrow \mathbb{R}$ we will denote $\bar{\psi}(t, x):=\psi(t, x, \bar{y}(t, x), \bar{u}(t, x))$ and given $u \in L^{\infty, \infty}(Q)$ we will write $\delta \psi(t, x):=\psi(t, x, \bar{y}(t, x), u(t, x))-\bar{\psi}(t, x)$. Similarly, for $\Psi: \Omega \times \mathbb{R} \rightarrow \mathbb{R}$ we set $\bar{\Psi}(x):=\Psi(x, \bar{y}(T, x))$.

Lemma 3.1. For $v \in L^{\infty, \infty}(Q)$ set $u:=\bar{u}+v$ and let us define $z_{1}[v] \in \mathcal{V}^{s}(Q)$ and $z_{2}[v] \in \mathcal{V}^{s}(Q)$ as the solutions of

$$
\begin{aligned}
\partial_{t} z_{1}-\Delta z_{1}+\bar{\varphi}_{y}(t, x) z_{1}+\delta \varphi(t, x) & =0 \text { in } Q, \\
z_{1} & =0 \text { in } \Sigma, \\
z_{1}(0, \cdot) & =0 \text { in } \Omega .
\end{aligned}
$$

and

$$
\begin{aligned}
\partial_{t} z_{2}-\Delta z_{2}+\bar{\varphi}_{y}(t, x) z_{2}+\frac{1}{2} \bar{\varphi}_{y y}(t, x) z_{1}[v]^{2}+\delta \varphi_{y}(t, x) z_{1}[v] & =0 \text { in } Q, \\
z_{2} & =0 \text { in } \Sigma, \\
z_{2}(0, \cdot) & =0 \text { in } \Omega,
\end{aligned}
$$

respectively. Then, setting $\delta y=y[u]-\bar{y}, d_{1}[v]=\delta y-z_{1}[v]$ and $d_{2}[v]=d_{1}[v]-z_{2}[v]$, the following estimates hold true

$$
\begin{gathered}
\left\|z_{1}[v]\right\| \mathcal{V}^{s}+\|\delta y\|_{\mathcal{V}^{s}}=O\left(\|v\|_{s, s}\right), \\
\left\|d_{1}[v]\right\|_{2,2}+\left\|d_{1}[v](T, \cdot)\right\|_{2}=O\left(\|\delta y\|_{\infty, \infty}\|v\|_{2,2}\right), \\
\left\|d_{2}[v]\right\|_{1,1}+\left\|d_{2}[v](T, \cdot)\right\|_{1}=O\left(\|\delta y\|_{\infty, \infty}\|v\|_{2,2}^{2}\right) .
\end{gathered}
$$

Proof. The proof is postponed to the Appendix.

Let us now fix some notations. Given $u \in L^{\infty, \infty}(Q)$ and $\lambda \in \mathbb{R}^{n_{E}+n_{I}}$ we write $\delta u:=u-\bar{u}$ and

$$
\begin{aligned}
& \bar{H}[\lambda](t, x):=H\left(t, x, \bar{y}(t, x), \bar{p}_{\lambda}(t, x), \bar{u}(t, x)\right), \quad \delta \bar{H}[\lambda](t, x):=H\left(t, x, \bar{y}(t, x), \bar{p}_{\lambda}(t, x), u(t, x)\right)-\bar{H}[\lambda](t, x), \\
& \bar{H}_{y}[\lambda](t, x):=H_{y}\left(t, x, \bar{y}(t, x), \bar{p}_{\lambda}(t, x), \bar{u}(t, x)\right), \quad \delta \bar{H}_{y}[\lambda](t, x):=H_{y}\left(t, x, \bar{y}(t, x), \bar{p}_{\lambda}(t, x), u(t, x)\right)-\bar{H}_{y}[\lambda](t, x),
\end{aligned}
$$

with similar notation for the second order derivatives. Using the estimates obtained in Lemma 3.1 and the definitions of the Hamiltonian and the adjoint state $\bar{p}_{\lambda}$, we can prove the following result. 
Proposition 3.2. (i) We have the first order expansion of the cost:

$$
\mathcal{L}(u, \lambda)-\mathcal{L}(\bar{u}, \lambda)=\int_{Q} \delta \bar{H}[\lambda](t, x) \mathrm{d} t \mathrm{~d} x+O\left(\|\delta y\|_{\infty, \infty}\|\delta u\|_{2,2}\right)
$$

where the O-term is uniform for $\lambda$ in a bounded set of $\mathbb{R}^{n_{E}+n_{I}}$.

(ii) We have the following second order expansion

$$
\begin{aligned}
\mathcal{L}(u, \lambda)-\mathcal{L}(\bar{u}, \lambda)= & \int_{Q}\left[\delta \bar{H}[\lambda](t, x)+\frac{1}{2} \bar{H}_{y y}[\lambda](t, x)\left(z_{1}[\delta u]\right)^{2}+\delta \bar{H}_{y}[\lambda](t, x) z_{1}[\delta u]\right] \mathrm{d} t \mathrm{~d} x \\
& +\int_{\Omega} \bar{\Phi}_{y y}[\lambda]\left(z_{1}[\delta u](T, x)\right)^{2} \mathrm{~d} x+O\left(\|\delta y\|_{\infty, \infty}\|\delta u\|_{2,2}^{2}\right)
\end{aligned}
$$

where the O-term is uniform for $\lambda$ in a bounded set of $\mathbb{R}^{n_{E}+n_{I}}$.

Proof. See the Appendix.

Given $v \in L^{2,2}(Q)$ define $\xi[v] \in \mathcal{V}^{2}(Q)$ as the unique solution of

$$
\begin{aligned}
\partial_{t} \xi-\Delta \xi+\bar{\varphi}_{y}(t, x) \xi+\bar{\varphi}_{u}(t, x) v & =0 \text { in } Q, \\
\xi & =0 \text { in } \Sigma, \\
\xi(0, \cdot) & =0 \text { in } \Omega .
\end{aligned}
$$

Given $\lambda \in \mathbb{R}^{n_{E}+n_{I}}$, let us define the linear and quadratic forms $Q_{1}[\bar{u}, \lambda]: L^{2,2}(Q) \rightarrow \mathbb{R}, Q_{2}[\bar{u}, \lambda]:$ $L^{2,2}(Q) \rightarrow \mathbb{R}$ as

$$
\begin{aligned}
Q_{1}[\bar{u}, \lambda] v & :=\int_{Q} \bar{H}_{u}[\lambda](t, x) v(t, x) \mathrm{d} t \mathrm{~d} x, \\
Q_{2}[\bar{u}, \lambda](v) & :=\int_{Q} \bar{H}_{(y, u)^{2}}[\lambda](t, x)(\xi[v], v)^{2} \mathrm{~d} t \mathrm{~d} x+\int_{\Omega} \bar{\Phi}_{y y}[\lambda](x)(\xi[v](T, x))^{2} \mathrm{~d} x .
\end{aligned}
$$

Now, let us consider a sequence $u_{k} \in L^{\infty, \infty}(Q)$ such that $\left\|u_{k}-\bar{u}\right\|_{2,2} \rightarrow 0$ and let $A_{k}$, $B_{k}$ be a sequence of measurable subsets of $[0, T] \times \Omega$ such that $\left|A_{k} \cup B_{k}\right|=T \times|\Omega|$ and $\left|B_{k}\right| \downarrow 0$ as $k \uparrow \infty$ (where we set $|\cdot|$ for the Lebesgue measure of a Lebesgue measurable set). Recalling (3.3), we set

$$
\begin{aligned}
& \delta_{k} u:=u_{k}-\bar{u}, \quad \delta_{A_{k}} u:=\mathbb{I}_{A_{k}}\left(u_{k}-\bar{u}\right), \quad \delta_{B_{k}} u:=\delta_{k} u-\delta_{A_{k}} u, \\
& z^{k}:=z_{1}\left[\delta_{k} u\right], \quad z^{A_{k}}:=z_{1}\left[\delta_{A_{k}} u\right] \quad \text { and } \quad z^{B_{k}}:=z_{1}\left[\delta_{B_{k}} u\right] .
\end{aligned}
$$

Similarly to (3.6), we write $\delta_{k} \bar{H}[\lambda](t, x)=H\left(t, x, \bar{y}(t, x), \bar{p}_{\lambda}(t, x), u_{k}(t, x)\right)-\bar{H}[\lambda](t, x)$, with an analogous definition for $\delta_{k} \bar{H}_{y}[\lambda](t, x)$. Using Proposition 3.2, we prove now the main result of this section, that will be fundamental in Sections 4 and 5 .

Theorem 3.3. Suppose that $\left\|\delta_{k} u\right\|_{2,2} \downarrow 0$ and that $\left\|\delta_{A_{k}} u\right\|_{\infty, \infty} \downarrow 0$. Then,

$$
\mathcal{L}\left(u_{k}, \lambda\right)-\mathcal{L}(\bar{u}, \lambda)=\int_{B_{k}} \delta_{k} \bar{H}[\lambda](t, x) \mathrm{d} t \mathrm{~d} x+Q_{1}[\bar{u}, \lambda] \delta_{A_{k}} u+\frac{1}{2} Q_{2}[\bar{u}, \lambda]\left(\delta_{A_{k}} u\right)+o\left(\left\|\delta_{k} u\right\|_{2}^{2}\right),
$$

where the o-term is uniform for $\lambda$ in a compact set of $\mathbb{R}^{n_{E}+n_{I}}$.

Proof. Let us first prove that

$$
\begin{aligned}
\mathcal{L}\left(u_{k}, \lambda\right)-\mathcal{L}(\bar{u}, \lambda)= & \int_{B_{k}} \delta_{k} \bar{H}[\lambda](t, x) \mathrm{d} t \mathrm{~d} x+\int_{Q}\left[\bar{H}_{u}[\lambda](t, x) \delta_{A_{k}} u+\frac{1}{2} \bar{H}_{(y, u)^{2}}[\lambda](t, x)\left(z^{A_{k}}, \delta_{A_{k}} u\right)^{2}\right] \mathrm{d} t \mathrm{~d} x \\
& +\int_{\Omega} \bar{\Phi}_{y y}[\lambda]\left(z^{A_{k}}(T, x)\right)^{2} \mathrm{~d} x+o\left(\left\|\delta_{k} u\right\|_{2}^{2}\right) .
\end{aligned}
$$


The proof is based on expansion (3.8). For notational simplicity we omit the dependence on $\lambda$ for $H$ and its derivatives. First note that, since $\left\|\delta_{A_{k}} u\right\|_{\infty, \infty} \downarrow 0$, a Taylor expansion implies that

$$
\begin{aligned}
\int_{A_{k}} \delta_{k} \bar{H}(t, x) \mathrm{d} t \mathrm{~d} x & =\int_{Q}\left[\bar{H}_{u}(t, x) \delta_{A_{k}} u+\frac{1}{2} \bar{H}_{u u}(t, x)\left(\delta_{A_{k}} u\right)^{2}\right] \mathrm{d} t \mathrm{~d} x+O\left(\left\|\delta_{A_{k}} u\right\|_{\infty, \infty}\left\|\delta_{A_{k}} u\right\|_{2,2}^{2}\right), \\
& =\int_{Q}\left[\bar{H}_{u}(t, x) \delta_{A_{k}} u+\frac{1}{2} \bar{H}_{u u}(t, x)\left(\delta_{A_{k}} u\right)^{2}\right] \mathrm{d} t \mathrm{~d} x+o\left(\left\|\delta_{k} u\right\|_{2,2}^{2}\right) .
\end{aligned}
$$

Thus, if

$$
\begin{aligned}
\int_{Q}\left[\frac{1}{2} \bar{H}_{y y}(t, x)\left(z^{k}\right)^{2}+\delta_{k} \bar{H}_{y} z^{k}\right] \mathrm{d} t \mathrm{~d} x & =\int_{Q}\left[\frac{1}{2} \bar{H}_{y y}(t, x)\left(z^{A_{k}}\right)^{2}+\bar{H}_{y u}(t, x) z^{A_{k}} \delta_{A_{k}} u\right] \mathrm{d} t \mathrm{~d} x+o\left(\left\|\delta_{k} u\right\|_{2}^{2}\right), \\
\int_{\Omega} \bar{\Phi}_{y y}[\lambda]\left(z^{k}(T, x)\right)^{2} \mathrm{~d} x & =\int_{\Omega} \bar{\Phi}_{y y}[\lambda]\left(z^{A_{k}}(T, x)\right)^{2} \mathrm{~d} x+o\left(\left\|\delta_{k} u\right\|_{2}^{2}\right)
\end{aligned}
$$

holds true, relation (3.13) follows from (3.8) and (3.15). Let us prove (3.15). Note that

$$
\int_{Q}\left[\frac{1}{2} \bar{H}_{y y}(t, x)\left(z^{k}\right)^{2}+\delta_{k} \bar{H}_{y}(t, x) z^{k}\right] \mathrm{d} t \mathrm{~d} x=\int_{Q}\left[\frac{1}{2} \bar{H}_{y y}(t, x)\left(z^{A_{k}}\right)^{2}+\delta_{k} \bar{H}_{y}(t, x) z^{A_{k}}\right] \mathrm{d} t \mathrm{~d} x+r_{k},
$$

where

$$
\left|r_{k}\right|=O\left(\int_{Q}\left[\left|\left(z^{A_{k}}\right)^{2}-\left(z^{k}\right)^{2}\right|+\left|\delta_{k} u\right|\left|z^{A_{k}}-z^{k}\right|\right] \mathrm{d} t \mathrm{~d} x\right)=O\left(\int_{Q}\left[\left|z^{B_{k}}\right|^{2}+\left|z^{B_{k}}\right|\left|z^{A_{k}}\right|+\left|\delta_{k} u\right|\left|z^{B_{k}}\right|\right] \mathrm{d} t \mathrm{~d} x\right) .
$$

By (2.3), there exists $\left.q_{1} \in\right] 1,2\left[\right.$ and $\left.q_{2} \in\right] 2, \infty\left[\right.$ such that $\left\|z^{B_{k}}\right\|_{2,2}=O\left(\left\|\delta_{B_{k}} u\right\|_{q_{1}, q_{1}}\right)$ and $\left\|z^{A_{k}}\right\|_{q_{2}, q_{2}}=$ $O\left(\left\|\delta_{A_{k}} u\right\|_{2,2}\right)=O\left(\left\|\delta_{k} u\right\|_{2,2}\right)$. By Hölder inequality (and setting $s^{*}:=s /(s-1)$ for $\left.s \in\right] 1, \infty[$ ) we get

$$
\left\|\delta_{B_{k}} u\right\|_{q_{1}, q_{1}}=\left(\int_{Q}\left|\delta_{B_{k}} u\right|^{q_{1}} \mathrm{~d} t \mathrm{~d} x\right)^{\frac{1}{q_{1}}} \leq\left(\int_{Q}\left(\mathbb{I}_{B_{k}}\right)^{\left(\frac{2}{q_{1}}\right)^{*}} \mathrm{~d} t \mathrm{~d} x\right)^{\frac{1}{\left(\frac{2}{q_{1}}\right)^{*}}}\left\|\delta_{k} u\right\|_{2,2}=o\left(\left\|\delta_{k} u\right\|_{2,2}\right) .
$$

Therefore, using Hölder inequality again, $r_{k}=o\left(\left\|\delta_{k} u\right\|_{2,2}\right)$ and so

$$
\int_{Q}\left[\frac{1}{2} \bar{H}_{y y}(t, x)\left(z^{k}\right)^{2}+\delta_{k} \bar{H}_{y}(t, x) z^{k}\right] \mathrm{d} t \mathrm{~d} x=\int_{Q}\left[\frac{1}{2} \bar{H}_{y y}(t, x)\left(z^{A_{k}}\right)^{2}+\delta_{k} \bar{H}_{y}(t, x) z^{A_{k}}\right] \mathrm{d} t \mathrm{~d} x+o\left(\left\|\delta_{k} u\right\|_{2,2}\right) .
$$

Now, since $\left\|\delta_{A_{k}} u\right\|_{\infty, \infty} \rightarrow 0$ we have that $\left\|z^{A_{k}}\right\|_{\infty, \infty} \rightarrow 0$ and so

$$
\begin{aligned}
\int_{Q} \delta_{k} \bar{H}_{y}(t, x) z^{A_{k}} \mathrm{~d} t \mathrm{~d} x & =\int_{Q} \bar{H}_{y u}(t, x) z^{A_{k}} \delta_{k} u \mathrm{~d} t \mathrm{~d} x+O\left(\int_{Q}\left|\delta_{k} u\right|^{2}\left|z^{A_{k}}\right| \mathrm{d} t \mathrm{~d} x\right) \\
& =\int_{Q} \bar{H}_{y u}(t, x) z^{A_{k}} \delta_{k} u \mathrm{~d} t \mathrm{~d} x+o\left(\left\|\delta_{k} u\right\|_{2,2}^{2}\right) .
\end{aligned}
$$

Noting that $\left.q_{2}^{*} \in\right] 1,2[$, we get

$$
\left|\int_{Q} \bar{H}_{y u}(t, x) z^{A_{k}} \delta_{B_{k}} u \mathrm{~d} t \mathrm{~d} x\right|=O\left(\left\|z^{A_{k}}\right\|_{q_{2}, q_{2}}\left\|\delta_{B_{k}} u\right\|_{q_{2}^{*}, q_{2}^{*}}\right)=o\left(\left\|\delta_{k} u\right\|_{2,2}^{2}\right),
$$

and so (3.19) yields

$$
\int_{Q} \delta_{k} \bar{H}_{y}(t, x) z^{A_{k}} \mathrm{~d} t \mathrm{~d} x=\int_{Q} \bar{H}_{y u}(t, x) z^{A_{k}} \delta_{A_{k}} u \mathrm{~d} t \mathrm{~d} x+o\left(\left\|\delta_{k} u\right\|_{2,2}^{2}\right) .
$$

Combining (3.18) and (3.20) gives the first identity in (3.15). In order to derive the second one, note that Fubini's theorem implies that

$$
\begin{aligned}
\int_{\Omega} \bar{\Phi}_{y y}[\lambda]\left(z^{k}(T, x)\right)^{2} \mathrm{~d} x & =2 \int_{\Omega} \bar{\Phi}_{y y}[\lambda]\left[\int_{0}^{T} z^{k}(t, x) \partial_{t} z^{k}(t, x) \mathrm{d} t\right] \mathrm{d} x \\
& =2 \int_{Q} \bar{\Phi}_{y y}[\lambda] z^{k}(t, x) \partial_{t} z^{k}(t, x) \mathrm{d} t \mathrm{~d} x
\end{aligned}
$$


Analogously,

$$
\int_{\Omega} \bar{\Phi}_{y y}[\lambda]\left(z^{A_{k}}(T, x)\right)^{2} \mathrm{~d} x=2 \int_{Q} \bar{\Phi}_{y y}[\lambda] z^{A_{k}}(t, x) \partial_{t} z^{A_{k}}(t, x) \mathrm{d} t \mathrm{~d} x
$$

Therefore, using

$$
z^{k} \partial_{t} z^{k}-z^{A_{k}} \partial_{t} z^{A_{k}}=\left(z^{k}-z^{A_{k}}\right) \partial_{t} z^{k}+\partial_{t}\left(z^{k}-z^{A_{k}}\right) z^{A_{k}}=z^{B_{k}} \partial_{t} z^{k}+\partial_{t} z^{B_{k}} z^{A_{k}}
$$

we obtain

$$
\left|\int_{\Omega}\left[\bar{\Phi}_{y y}[\lambda]\left(z^{k}(T, x)\right)^{2}-\bar{\Phi}_{y y}[\lambda]\left(z^{A_{k}}(T, x)\right)^{2}\right] \mathrm{d} x\right|=O\left(\int_{Q}\left[\left|z^{B_{k}}\right|\left|\partial_{t} z^{k}\right|+\left|\partial_{t} z^{B_{k}}\right|\left|z^{A_{k}}\right|\right] \mathrm{d} t \mathrm{~d} x\right) .
$$

By considering $\left.q_{1} \in\right] 1,2\left[\right.$ and $\left.q_{2} \in\right] 2, \infty[$ as above, Hölder inequality implies that

$$
\begin{aligned}
\left|\int_{\Omega}\left[\bar{\Phi}_{y y}[\lambda]\left(z^{k}(T, x)\right)^{2}-\bar{\Phi}_{y y}[\lambda]\left(z^{A_{k}}(T, x)\right)^{2}\right] \mathrm{d} x\right| & =O\left(\left\|z^{B_{k}}\right\|_{2,2}\left\|\partial_{t} z^{k}\right\|_{2,2}+\left\|\partial_{t} z^{B_{k}}\right\|_{q_{2}^{*}, q_{2}^{*}}\left\|z^{A_{k}}\right\|_{q_{2}, q_{2}}\right), \\
& =o\left(\left\|\delta_{k} u\right\|_{2,2}^{2}\right),
\end{aligned}
$$

where we have used (2.5) in order to estimate $\left\|\partial_{t} z^{k}\right\|_{2,2}=O\left(\left\|\delta_{k} u\right\|_{2,2}\right)$ and $\left\|\partial_{t} z^{B_{k}}\right\|_{q_{2}^{*}, q_{2}^{*}}=O\left(\left\|\delta_{B_{k}} u\right\|_{q_{2}^{*}, q_{2}^{*}}\right)=$ $o\left(\left\|\delta_{k} u\right\|_{2,2}\right)$. Expansion (3.13) follows. Now, defining $\zeta^{k}:=z^{A_{k}}-\xi^{k}$, where $\xi^{k}:=\xi\left[\delta_{A_{k}} u\right]$ (recall $(3.9))$, we have that

$$
\begin{aligned}
\partial_{t} \zeta^{k}-\Delta \zeta^{k}+\bar{\varphi}_{y}(t, x) \zeta^{k} & =O\left(\left|\delta_{A_{k}} u\right|^{2}\right) \text { in } Q, \\
\zeta^{k} & =0 \text { in } \Sigma, \\
\zeta^{k}(0, \cdot) & =0 \text { in } \Omega .
\end{aligned}
$$

By (2.5), we obtain that

$$
\left\|\zeta^{k}\right\|_{2,2}+\left\|\zeta^{k}(T, \cdot)\right\|_{2}=O\left(\left(\int_{Q}\left|\delta_{A_{k}} u\right|^{4} \mathrm{~d} t \mathrm{~d} x\right)^{\frac{1}{2}}\right)=O\left(\left\|\delta_{A_{k}} u\right\|_{\infty, \infty}\left\|\delta_{A_{k}} u\right\|_{2,2}\right)=o\left(\left\|\delta_{A_{k}} u\right\|_{2,2}\right) .
$$

Using this estimate, it is straightforward to obtain (3.12) from (3.13).

Remark 3.4. When $\|\delta u\|_{2,2}$ is small, (3.12) provides a second order expansion of $\mathcal{L}(u, \lambda)-\mathcal{L}(\bar{u}, \lambda)$ that is decomposed in two principal terms. The first one corresponds to a classical "weak" second order expansion (see [26]) on $\delta u \mathbb{I}_{A}\left(\mathbb{I}_{A}\right.$ denotes the indicator function of the set $A$ ) with $A$ being a measurable set such that $\left\|\delta u \mathbb{I}_{A}\right\|_{\infty, \infty}$ is small. The second term in the expansion takes into account large deviations of $u(t, x)$ around $\bar{u}(t, x)$, but over a set of small measure.

\section{Second order analysis for strong solutions}

In this section we study second order optimality conditions for strong local solutions. Let us first recall that given a Banach space $\left(X,\|\cdot\|_{X}\right)$ and $K \subseteq X$, the tangent cone to $K$ at $u$ is defined as

$$
T_{K}(u):=\left\{v \in X \mid \exists u(\sigma)=u+\sigma v+o(\sigma) \in K, \tau>0,\|o(\sigma) / \sigma\|_{X} \rightarrow 0, \text { as } \sigma \downarrow 0\right\} .
$$

Definition 4.1. (i) We say that $\lambda=\left(\lambda_{E}, \lambda_{I}\right) \in \mathbb{R}^{n_{E}+n_{I}}$ is a Lagrange multiplier at $\bar{u} \in \mathcal{K}$ if

$$
Q_{1}[\bar{u}, \lambda] v \geq 0 \text { for all } v \in T_{\mathcal{K}_{1}}(\bar{u}), \lambda_{I}^{j} \geq 0, \lambda_{I}^{j} G_{I}^{j}(\bar{u})=0, \text { for all } j=1, \ldots, n_{I} .
$$

The set of Lagrange multipliers at $\bar{u}$ is denoted as $\Lambda_{L}(\bar{u})$.

(ii) We say that $\lambda=\left(\lambda_{E}, \lambda_{I}\right) \in \mathbb{R}^{n_{E}+n_{I}}$ is a Pontryagin multiplier at $\bar{u} \in \mathcal{K}$ if $\lambda \in \Lambda_{L}(\bar{u})$ and a.e. in $Q$

$$
\bar{H}[\lambda](t, x) \leq H\left(t, x, \bar{y}, \bar{p}_{\lambda}, u\right) \quad \forall u \in[a(t, x), b(t, x)] .
$$

The set of Pontryagin multipliers at $\bar{u}$ is denoted as $\Lambda_{P}(\bar{u})$. 
Let us define the active sets

$$
\begin{gathered}
A_{a}(\bar{u}):=\{(t, x) \in Q ; \bar{u}(t, x)=a(t, x)\}, A_{b}(\bar{u}):=\{(t, x) \in Q ; \bar{u}(t, x)=b(t, x)\}, \\
I(\bar{u}):=\left\{j \in\left\{1, \ldots, n_{I}\right\} ; G_{I}^{j}(\bar{u})=0\right\} .
\end{gathered}
$$

If Robinson's constraint qualification condition (see [24])

$$
0 \in \operatorname{int}\left\{G(\bar{u})+D G(\bar{u})\left(\mathcal{K}_{1}-\bar{u}\right)-\mathcal{K}_{2}\right\}
$$

holds true, then by [26, Lemma 4.2$]$

$$
T_{\mathcal{K}}(\bar{u}):=\left\{v \in L^{2,2}(Q) ; v \geq 0 \text { in } A_{a}(\bar{u}) \text { and } v \leq 0 \text { in } A_{b}(\bar{u}), D G_{E}(\bar{u}) v=0, D G_{I}^{i}(\bar{u}) v \leq 0 \forall i \in I(\bar{u})\right\} .
$$

Moreover, as a consequence of [14, Corollary 2.2], at any weak solution $\bar{u}$ of $(C P)$ we have that $\Lambda_{L}(\bar{u})$ is a nonempty compact subset of $\mathbb{R}^{n_{E}+n_{I}}$.

The critical cone $C_{\mathcal{K}}(\bar{u})$ to $\mathcal{K}$ at $\bar{u}$ is defined as

$$
C_{\mathcal{K}}(\bar{u}):=\left\{v \in T_{\mathcal{K}}(\bar{u}) ; D J(\bar{u}) v=0\right\} .
$$

In order to provide a second order necessary condition we will assume the following constraint qualification

(H3) There exists $\lambda \in \Lambda_{L}(\bar{u})$ such that

$$
0 \in \operatorname{int}\left(D G(\bar{u})\left[\left(\mathcal{K}_{1}-\bar{u}\right) \cap\left(\bar{H}_{u}[\lambda]\right)^{\perp}\right]-T_{\mathcal{K}_{2}}(G(\bar{u})) \cap \lambda^{\perp}\right)
$$

where $\left(\bar{H}_{u}[\lambda]\right)^{\perp}$ denotes the functions $v \in L^{2,2}(Q)$ such that $\int_{Q} \bar{H}_{u}[\lambda](t, x) v(t, x) \mathrm{d} t \mathrm{~d} x=0$ and $\lambda^{\perp}$ is the subspace of $\mathbb{R}^{n_{E}} \times \mathbb{R}^{n_{I}}$ which is orthogonal to $\{\lambda\}$.

Remark 4.2. (i) Condition (4.6), introduced by Shapiro in [25] for general optimization problems and in [11] in the context of semilinear elliptic equations with finitely many state constraints, implies $(R C Q)$ and the fact that $\Lambda_{L}(\bar{u})$ is a singleton (see [26, Lemma 5.4]). This qualification condition is implied by the classical surjectivity type assumption for similar problems in the context of semilinear elliptic equations (see e.g. [13, Condition (3.1)] and [26, Remark 5.3 (ii)]).

(ii) Under (4.6), second order necessary conditions for local weak minima are proven in [26, Theorem 5.7]. Of course, if no final state constraints are considered, then such qualification condition is not necessary to establish second order necessary conditions.

We say that the quadratic growth condition for the Hamiltonian holds true at $(\bar{u}, \lambda) \in \mathcal{K} \times \Lambda_{P}(\bar{u})$ if there exists $\alpha>0$ such that a.e. in $Q$

$$
\bar{H}[\lambda](t, x)+\frac{\alpha}{2}|u-\bar{u}(t, x)|^{2} \leq H\left(t, x, \bar{y}, \bar{p}_{\lambda}, u\right) \quad \forall u \in[a(t, x), b(t, x)] .
$$

Let us provide a second order necessary condition for quadratic growth in the $L^{s}$-sense. Since any strong local solution satisfying the quadratic growth condition is a $L^{s}$-weak local solution satisfying the quadratic growth condition, the following result is also verified for strong solutions.

Theorem 4.3. Suppose that (H1)-(H3) hold true. Then, if $s \in\left[1, \infty\left[\right.\right.$ and $\bar{u}$ is a $L^{s}$-weak local solution of $(C P)$ satisfying the quadratic growth condition (2.14), we have that:

(i) There exists $\lambda \in \mathbb{R}^{n_{E}+n_{I}}$ such that $\Lambda_{L}(\bar{u})=\Lambda_{P}(\bar{u})=\{\lambda\}$.

(i) Condition (4.7) holds true.

(ii) There exists $\alpha>0$ such that for every $v \in C_{\mathcal{K}}(\bar{u})$ we have that $Q_{2}[\bar{u}, \lambda](v) \geq \alpha\|v\|_{2,2}^{2}$ 
Proof. If $\bar{u}$ is a $L^{s}$ weak local solution of $(C P)$ satisfying (2.14) then in particular $\bar{u}$ satisfies (2.12) and so the existence of $\lambda \in \mathbb{R}^{n_{E}+n_{I}}$ such that $\Lambda_{L}(\bar{u})=\{\lambda\}$ and (iii) follow from [26, Theorem 5.7]. The existence of $\lambda^{\prime} \in \mathbb{R}^{n_{E}+n_{I}}$ such that condition (4.7) is verified, follows by Pontryagin's principle (see $[18,12,14])$ applied to the optimal control problem $\inf \left\{J(u)-\frac{1}{2} \alpha\|u-\bar{u}\|_{2,2}^{2}, \quad u \in \mathcal{K}\right\}$. Noting that the latter problem has $\Lambda_{L}(\bar{u})$ as Lagrange multiplier set we get that $\lambda=\lambda^{\prime}$ from which the result follows.

Recall that if $\mathcal{H}$ is a Hilbert space, a quadratic form $Q: \mathcal{H} \rightarrow \mathbb{R}$ is a Legendre form if it satisfies: (a) $Q$ is weakly lower semicontinuous.

(b) If $v_{k} \in \mathcal{H}$ converges weakly to $v$ and $Q\left(v_{k}\right)$ converges to $Q(v)$, then $v_{k}$ converges strongly to $v$.

Now, we prove our main result which is a sufficient condition for the strong quadratic growth property. Note that in contrast to the necessary condition in Theorem 4.3, no qualification condition is assumed.

Theorem 4.4. Suppose that (H1)-(H2) hold true and that:

(i) $\Lambda_{P}(\bar{u}) \neq \emptyset$.

(ii) There exists $\bar{\lambda} \in \Lambda_{P}(\bar{u})$ such that the quadratic growth condition for the Hamiltonian holds true at $(\bar{u}, \bar{\lambda})$.

(iii) For all $\lambda \in \Lambda_{P}(\bar{u})$ the quadratic form $Q[\bar{u}, \lambda]$ is a Legendre form and there exists $\alpha>0$ such that

$$
\max _{\lambda \in \Lambda_{P}(\bar{u})} Q[\bar{u}, \lambda](v) \geq \alpha\|v\|_{2,2}^{2} \quad \forall v \in C_{\mathcal{K}}(\bar{u}) .
$$

Then, $\bar{u}$ is a strong local solution of $(C P)$ satisfying the quadratic growth condition.

Proof. If (2.16) does not hold, then there exists a sequence $u_{k} \neq \bar{u} \in \mathcal{K}$, with associated states $y_{k}:=y\left[u_{k}\right]$, such that, setting $\delta_{k} u:=u_{k}-\bar{u}$ and $\delta_{k} y:=y_{k}-\bar{y}$, one has

$$
J\left(u_{k}\right)-J(\bar{u}) \leq o\left(\left\|\delta_{k} u\right\|_{2,2}^{2}\right) \quad \text { as } \quad\left\|\delta_{k} y\right\|_{\infty, \infty} \rightarrow 0 .
$$

By definition, for every $\lambda \in \Lambda_{L}(\bar{u})$ we have that $J\left(u_{k}\right) \geq \mathcal{L}\left(u_{k}, \lambda\right)$ and $J(\bar{u})=\mathcal{L}(\bar{u}, \lambda)$, therefore by Proposition 3.2(i) we have that

$$
\int_{Q} \delta_{k} \bar{H}[\lambda](t, x) \mathrm{d} t \mathrm{~d} x+O\left(\left\|\delta_{k} u\right\|_{2,2}\left\|\delta_{k} y\right\|_{\infty, \infty}\right)=\mathcal{L}\left(u_{k}, \lambda\right)-\mathcal{L}(\bar{u}, \lambda) \leq o\left(\left\|\delta_{k} u\right\|_{2,2}^{2}\right) .
$$

Setting $\lambda=\bar{\lambda}$, assumption (ii) implies that $\left\|\delta_{k} u\right\|_{2,2} \rightarrow 0$. Now, let us define

$$
A_{k}:=\left\{(t, x) \in Q ;|u(t, x)-\bar{u}(t, x)| \leq \sqrt{\left\|\delta_{k} u\right\|_{1,1}}\right\}, \quad B_{k}:=Q \backslash A_{k},
$$

and $\delta_{A_{k}} u$ and $\delta_{B_{k}} u$ as in (3.11). Since $\left\|\delta_{k} u\right\|_{2,2} \rightarrow 0$, we obtain that $\left\|\delta_{A_{k}} u\right\|_{\infty, \infty} \rightarrow 0$ and using the Chebyshev inequality we get that $\left|B_{k}\right| \rightarrow 0$. Therefore, by (3.12) and (4.8), for every $\lambda \in \Lambda_{L}(\bar{u})$ we have that

$$
\int_{B_{k}} \delta_{k} \bar{H}[\lambda](t, x) \mathrm{d} t \mathrm{~d} x+Q_{1}[\bar{u}, \lambda]\left(\delta_{A_{k}} u\right)+\frac{1}{2} Q_{2}[\bar{u}, \lambda]\left(\delta_{A_{k}} u\right) \leq o\left(\left\|\delta_{k} u\right\|_{2,2}^{2}\right) .
$$

Suppose that $\left\|\delta_{A_{k}} u\right\|_{2,2}=o\left(\left\|\delta_{B_{k}} u\right\|_{2,2}\right)$. Then we have that $\left\|\delta_{k} u\right\|_{2,2}=O\left(\left\|\delta_{B_{k}} u\right\|_{2,2}\right)$. Since $\delta_{A_{k}} u \in$ $T_{\mathcal{K}_{1}}(\bar{u})$, for every $\lambda \in \Lambda_{L}(\bar{u})$ we have that $Q_{1}[\bar{u}, \lambda] \delta_{A_{k}} u \geq 0$. Also, by the continuity of the quadratic form $Q_{2}[\bar{u}, \lambda](\cdot)$ we get that $Q_{2}[\bar{u}, \lambda]\left(\delta_{A_{k}} u\right)=O\left(\left\|\delta_{A_{k}} u\right\|_{2,2}^{2}\right)$, and so, using (4.9), we obtain the inequality

$$
\int_{B_{k}} \delta_{k} \bar{H}[\lambda](t, x) \mathrm{d} t \mathrm{~d} x \leq o\left(\left\|\delta_{k} u\right\|_{2,2}^{2}\right)+O\left(\left\|\delta_{A_{k}} u\right\|_{2,2}^{2}\right)=o\left(\left\|\delta_{B_{k}} u\right\|_{2,2}^{2}\right) .
$$


Setting $\lambda=\bar{\lambda}$ in the above inequality, by condition (ii) we get that $\alpha\left\|\delta_{B_{k}} u\right\|_{2,2}^{2} \leq o\left(\left\|\delta_{B_{k}} u\right\|_{2,2}^{2}\right.$ ), for some $\alpha>0$, which is impossible. Thus, except for some subsequence, $\left\|\delta_{B_{k}} u\right\|_{2,2}=O\left(\left\|\delta_{A_{k}} u\right\|_{2,2}\right)$. Now, let us set $\sigma_{k}:=\left\|\delta_{A_{k}} u\right\|_{2,2}$, which can be assumed to be nonzero, and define $v_{k}:=\delta_{A_{k}} u / \sigma_{k}$. Since $\left\|v_{k}\right\|_{2,2}=1$, we have that, up to some subsequence, $v_{k}$ converges weakly in $L^{2,2}(Q)$ to some $\bar{v}$. Note that since $v^{k} \in T_{\mathcal{K}_{1}}(\bar{u})$ and $T_{\mathcal{K}_{1}}(\bar{u})$ is closed and convex in $L^{2,2}(Q)$, we obtain that $\bar{v} \in T_{\mathcal{K}_{1}}(\bar{u})$. Let us now prove that $\bar{v} \in C_{\mathcal{K}}(\bar{u})$. First note that, by the results in [26, Section 3],

$$
J\left(u_{k}\right)-J(\bar{u})=D J(\bar{u}) \delta_{k} u+O\left(\left\|\delta_{k} u\right\|_{2,2}^{2}\right)=D J(\bar{u}) \delta_{k} u+o\left(\left\|\delta_{k} u\right\|_{2,2}\right),
$$

and, recalling (3.9) and using [26, Remark 3.4],

$$
D J(\bar{u}) \delta_{k} u=\int_{Q}\left[\bar{\ell}_{y}(t, x) \zeta\left[\delta_{k} u\right]+\bar{\ell}_{u}(t, x) \delta_{k} u\right] \mathrm{d} t \mathrm{~d} x+\int_{\Omega} \bar{\Phi}_{y}(x) \zeta\left[\delta_{k} u\right](T, x) \mathrm{d} x .
$$

On the other hand, estimate (2.6) implies that

$$
\left\|\zeta\left[\delta_{B_{k}} u\right]\right\|_{1,1}+\left\|\zeta\left[\delta_{B_{k}} u\right](T, \cdot)\right\|_{1}=O\left(\left\|\delta_{B_{k}} u\right\|_{1,1}\right)=o\left(\left\|\delta_{k} u\right\|_{2,2}\right) .
$$

Therefore, since $\left\|\delta_{k} u\right\|_{2,2}=O\left(\left\|\delta_{A_{k}} u\right\|_{2,2}\right)$, equations (4.8), (4.11) and (4.12), yield

$$
\int_{Q}\left[\bar{\ell}_{y}(t, x) \zeta\left[\delta_{A_{k}} u\right]+\bar{\ell}_{u}(t, x) \delta_{A_{k}} u\right] \mathrm{d} t \mathrm{~d} x+\int_{\Omega} \bar{\Phi}_{y}(x) \zeta\left[\delta_{A_{k}} u\right](T, x) \mathrm{d} x \leq o\left(\left\|\delta_{A_{k}} u\right\|_{2,2}\right) .
$$

Dividing by $\sigma_{k}$ we get that

$$
\int_{Q}\left[\bar{\ell}_{y}(t, x) \zeta\left[v_{k}\right]+\bar{\ell}_{u}(t, x) v_{k}\right] \mathrm{d} t \mathrm{~d} x+\int_{\Omega} \bar{\Phi}_{y}(x) \zeta\left[v_{k}\right](T, x) \mathrm{d} x \leq o(1) .
$$

Using [26, Proposition 2.1(iii)] and passing to the limit we obtain that $D J(\bar{u}) \bar{v} \leq 0$. On the other hand, the same type of computations implies that

$$
\int_{\Omega}\left(\bar{\Phi}_{E}\right)_{y}(x) \zeta[\bar{v}](T, x) \mathrm{d} x=0 \quad \text { and } \int_{\Omega}\left(\bar{\Phi}_{I}\right)^{j}(x) \zeta[\bar{v}](T, x) \mathrm{d} x \leq 0, \quad \forall j \in I(\bar{u}),
$$

which together with the fact that $\lambda \in \Lambda_{L}(\bar{u})$ and $\bar{v} \in T_{\mathcal{K}_{1}}(\bar{u})$, implies that $D J(u) \bar{v}=0$. Therefore, we have proven that $\bar{v} \in C_{\mathcal{K}}(\bar{u})$. Since for all $\lambda \in \Lambda_{P}(\bar{u})$ one has

$$
\int_{B_{k}} \delta_{k} H[\bar{u}, \lambda](t, x) \mathrm{d} t \mathrm{~d} x+Q_{1}[\bar{u}, \lambda]\left(\delta_{A_{k}} u\right) \geq 0
$$

we get with (4.9) that

$$
Q_{2}[\bar{u}, \lambda]\left(v_{k}\right) \leq o(1) .
$$

By assumption (iii), there exists $\lambda_{\bar{v}} \in \Lambda_{P}(\bar{u})$ such that $Q_{2}\left[\bar{u}, \lambda_{\bar{v}}\right](\bar{v}) \geq \alpha\|\bar{v}\|_{2,2}^{2}$. Since $Q_{2}\left[\bar{u}, \lambda_{\bar{v}}\right]$ is a Legendre form, (4.13) yields,

$$
0 \leq \alpha\|\bar{v}\|_{2,2}^{2} \leq Q_{2}\left[\bar{u}, \lambda_{\bar{v}}\right](\bar{v}) \leq \liminf _{k \rightarrow \infty} Q_{2}\left[\bar{u}, \lambda_{\bar{v}}\right]\left(v_{k}\right) \leq \limsup _{k \rightarrow \infty} Q_{2}\left[\bar{u}, \lambda_{\bar{v}}\right]\left(v_{k}\right) \leq 0,
$$

and so $\bar{v}=0$ and $Q_{2}\left[\bar{u}, \lambda_{\bar{v}}\right](\bar{v})=0=\lim _{k \rightarrow \infty} Q_{2}\left[\bar{u}, \lambda_{\bar{v}}\right]\left(v_{k}\right)$, which is imposible because the fact that $Q_{2}\left[\bar{u}, \lambda_{\bar{v}}\right]$ is a Legendre form yields that $v_{k} \rightarrow \bar{v}$ strongly in $L^{2,2}(Q)$, contradicting $\left\|v_{k}\right\|_{2,2}=1$. 
In the case when no final constraints are considered, i.e. when we consider the problem $\left(C P^{\prime}\right)$, we can prove a characterization of quadratic growth in the strong sense without the Legendre form assumption for the quadratic form. We notice that in this case the critical cone is given by

$$
C_{\mathcal{K}_{1}}(\bar{u})=\left\{v \in L^{2,2}(Q) ; v \geq 0 \text { in } A_{a}(\bar{u}) \text { and } v \leq 0 \text { in } A_{b}(\bar{u}), \bar{H}_{u}(t, x) v(t, x)=0 \text { a.e. in } Q\right\} .
$$

Since there is no dependence in $\lambda$, we denote by $\bar{p}$ the unique adjoint state associated with $\bar{u}$ and $\bar{H}(t, x):=H(t, x, \bar{y}(t, x), \bar{p}(t, x), \bar{u}(t, x))$, with similar notations for the derivatives of $H$ evaluated at $(t, x, \bar{y}(t, x), \bar{p}(t, x), \bar{u}(t, x))$. For $v \in L^{2,2}(Q)$, we set

$$
\begin{aligned}
Q_{1}[\bar{u}] v & :=\int_{Q} \bar{H}_{u}(t, x) v(t, x) \mathrm{d} t \mathrm{~d} x, \\
Q_{2}[\bar{u}](v) & :=\int_{Q} \bar{H}_{(y, u)^{2}}(t, x)(\xi[v], v)^{2} \mathrm{~d} t \mathrm{~d} x+\int_{\Omega} \bar{\Phi}_{y y}(x)(\xi[v](T, x))^{2} \mathrm{~d} x,
\end{aligned}
$$

where $\xi[v]$ is defined by $(3.9)$.

Theorem 4.5. Assume (H1)-(H2). Then, the following assertions are equivalent:

(i) The control $\bar{u}$ is a strong local solution of $\left(C P^{\prime}\right)$ satisfying the quadratic growth condition.

(ii) There exists $\alpha>0$ such that the following conditions hold true:

(ii.1) For almost all $(t, x) \in Q$ we have that

$$
\bar{H}(t, x)+\frac{\alpha}{2}|u-\bar{u}(t, x)|^{2} \leq H(t, x, \bar{y}(t, x), \bar{p}(t, x), u) \quad \forall u \in[a(t, x), b(t, x)] .
$$

(ii.2) For all $v \in C_{\mathcal{K}_{1}}(\bar{u})$ it holds that $Q_{2}[\bar{u}] v^{2} \geq \alpha\|v\|_{2,2}^{2}$.

Proof. The proof is similar to the proof of [3, Theorem 4.24]. The fact that (i) $\Rightarrow$ (ii) follows by the same argument than the one in the proof of Theorem 4.3. Note that, since we are not considering final state constraints, the qualification condition (4.6) is not needed (see [26]). As in the proof of Theorem 4.4, if (2.16) (with $\mathcal{K}$ replaced by $\mathcal{K}_{1}$ ) does not hold, then there exists a sequence $u_{k} \in \mathcal{K}_{1}$ satisfying that $\left\|y\left[u_{k}\right]-\bar{y}\right\|_{\infty, \infty} \rightarrow 0$ and (setting $\delta_{k} u:=u_{k}-\bar{u}$ )

$$
J\left(u_{k}\right)-J(\bar{u}) \leq o\left(\left\|\delta_{k} u\right\|_{2,2}^{2}\right) \quad \text { as } k \uparrow \infty .
$$

By (3.7) with $\lambda=0$ and (4.15) we get (setting $\delta_{k} y:=y\left[u_{k}\right]-\bar{y}$ )

$$
\frac{\alpha}{2}\left\|\delta_{k} u\right\|_{2}^{2} \leq o\left(\left\|\delta_{k} u\right\|_{2,2}^{2}\right)+O\left(\left\|\delta_{k} y\right\|_{\infty, \infty}\left\|\delta_{k} u\right\|_{2,2}\right)=o(1),
$$

which implies that $u_{k} \rightarrow \bar{u} \in L^{2,2}(Q)$. Now, for a.a. $(t, x) \in Q$, let us set

$$
\kappa_{t, x}:= \begin{cases}1 & \text { if } \bar{H}_{u}(t, x)=0, \\ 1 /\left|\bar{H}_{u}(t, x)\right| & \text { otherwise }\end{cases}
$$

and $B_{k}:=B_{k}^{1} \cup B_{k}^{2}$, where

$$
B_{k}^{1}:=\left\{(t, x) \in Q ;\left|\delta_{k} u(t, x)\right| \geq \sqrt{\left\|\delta_{k} u\right\|_{2,2}}\right\}, B_{k}^{2}:=\left\{(t, x) \in Q ; \kappa_{t, x} \geq 1 /\left\|\delta_{k} u\right\|_{2,2}^{1 / 4}\right\} .
$$

By the Chebyshev inequality $\left|B_{k}^{1}\right| \rightarrow 0$ as $k \uparrow \infty$. By the dominated convergence theorem, we also have that $\left|B_{k}^{2}\right|=\int_{Q} I_{\left\{(t, x) \in B_{k}^{2}\right\}} \mathrm{d} t \mathrm{~d} x \rightarrow 0$, which implies that $\left|B_{k}\right| \rightarrow 0$. Defining $A_{k}:=Q \backslash B_{k}$ and decomposing $\delta_{k} u=\delta_{A_{k}} u+\delta_{B_{k}} u$, we obtain that $\left\|\delta_{A_{k}} u\right\|_{\infty, \infty} \rightarrow 0$. Thus, letting $\lambda=0$ in (3.12), we get

$$
J\left(u_{k}\right)-J(\bar{u})=\int_{B_{k}} \delta_{k} \bar{H}(t, x) \mathrm{d} t \mathrm{~d} x+Q_{1}[\bar{u}] \delta_{A_{k}} u+\frac{1}{2} Q_{2}[\bar{u}]\left(\delta_{A_{k}} u\right)+o\left(\left\|\delta_{k} u\right\|_{2,2}^{2}\right) .
$$


Now, set $\sigma_{A_{k}}:=\left\|\delta_{A_{k}} u\right\|_{2,2}$ and $\sigma_{B_{k}}:=\left\|\delta_{B_{k}} u\right\|_{2,2}$, which yields $\left\|\delta_{k} u\right\|_{2,2}^{2}=\sigma_{A_{k}}^{2}+\sigma_{B_{k}}^{2}$. If $\sigma_{A_{k}}=o\left(\sigma_{B_{k}}\right)$, then (4.17) and the continuity of the quadratic form $Q_{2}[\bar{u}]$ imply that

$$
\int_{B_{k}} \delta \bar{H}(t, x) \mathrm{d} t \mathrm{~d} x+Q_{1}[\bar{u}] \delta_{A_{k}} u \leq o\left(\sigma_{B_{k}}^{2}\right) .
$$

Since $Q_{1}[\bar{u}] \delta_{A_{k}} u \geq 0$, (4.15) would yield that $\frac{\alpha}{2} \sigma_{B_{k}}^{2} \leq o\left(\sigma_{B_{k}}^{2}\right)$, which is impossible. Therefore, we must have, except for some subsequence, that $\sigma_{B_{k}}=O\left(\sigma_{A_{k}}\right)$. Let us define $v_{k}:=\delta_{A_{k}} u / \sigma_{A_{k}}$. By (4.15) we have $\int_{B_{k}} \delta \bar{H}(t, x) \mathrm{d} t \mathrm{~d} x \geq 0$ and $Q_{1}[\bar{u}] \delta_{A_{k}} u \geq 0$. Therefore, (4.16) and (4.17) imply that

$$
Q_{2}[\bar{u}]\left(v_{k}\right) \leq o(1) .
$$

Note that if $v_{k} \in C_{\mathcal{K}_{1}}(\bar{u})$ then (4.18) would yield a contradiction with (ii.2). The problem is that a priori $v_{k}$ belongs only to $T_{\mathcal{K}_{1}}(\bar{u})$. The idea is to decompose $v_{k}=v_{k, 1}+v_{k, 2}$ where $v_{k, 1} \in C_{\mathcal{K}_{1}}(\bar{u})$ and prove that $\left\|v_{k, 2}\right\|_{2,2} \rightarrow 0$, which together with (4.18) and (ii.2) would yield the desired contradiction. In order to construct such decomposition, first notice that $C_{\mathcal{K}_{1}}(\bar{u})=\left\{v \in L^{2,2}(Q) ; v(t, x) \in\right.$ $C_{t, x}$ for a.a. $\left.(t, x) \in Q\right\}$, where

$$
C_{t, x}:=\left\{v \in \mathbb{R} ; \bar{H}_{u}(t, x) v=0, v \geq 0 \text { if }(t, x) \in A_{a}(\bar{u}) \text { and } v \leq 0 \text { if }(t, x) \in A_{b}(\bar{u})\right\} .
$$

Thus, it is natural to define $v_{k, 1}(t, x):=P_{C_{t, x}}\left(v_{k}(t, x)\right)$ (where $P$. denotes the projection operator), which is measurable in terms of $(t, x)$ and $(t, x) \rightarrow v_{k, 1}(t, x) \in C_{\mathcal{K}_{1}}(\bar{u})$. Using that for every $h \in \mathbb{R}$ we have, denoting $d\left(h, C_{t, x}\right):=\inf \left\{|z-h| ; z \in C_{t, x}\right\}$, and $(h)_{+}:=\max \{h, 0\}$

$$
d\left(h, C_{t, x}\right) \leq \kappa_{t, x}\left(\left|\bar{H}_{u}(t, x) h\right|+\mathbb{I}_{A_{a}}(t, x)(-h)_{+}+\mathbb{I}_{A_{b}}(t, x)(h)_{+}\right),
$$

by definition of $v_{k, 1}$ and the fact that $v_{k} \in T_{\mathcal{K}_{1}}(\bar{u})$, we obtain that

$$
\left|v_{k, 2}(t, x)\right|=d\left(v_{k}(t, x), C_{t, x}\right) \leq \kappa_{t, x}\left|\bar{H}_{u}(t, x) v_{k}(t, x)\right| \leq \frac{1}{\left\|\delta_{k} u\right\|_{2,2}^{1 / 4}} \bar{H}_{u}(t, x) v_{k}(t, x),
$$

where we have used that $\bar{H}_{u}(t, x) v_{k}(t, x) \geq 0$ and that $v_{k} \equiv 0$ on $B_{k}$. Now, by the continuity of the quadratic form $Q_{2}[\bar{u}]$, we have that (4.17) implies that

$$
\int_{A_{k}} \bar{H}_{u}(t, x) v_{k}(t, x) \mathrm{d} t \mathrm{~d} x \leq O\left(\sigma_{A_{k}}\right)
$$

which together with (4.20) implies that $\left\|v_{k, 2}\right\|_{1,1} \leq O\left(\sigma_{A_{k}} /\left\|\delta_{k} u\right\|_{2,2}^{1 / 4}\right)$. On the other hand, by definition of $v_{k}^{1}(t, x)$ and $C_{t, x}$ we have that $\left|v_{k, 2}(t, x)\right| \leq\left|v_{k}(t, x)\right|$. And so, since $\left\|v_{k}\right\|_{\infty, \infty} \leq \frac{\sqrt{\left\|\delta_{k} u\right\|_{2,2}}}{\sigma_{A_{k}}}$ by definition of the set $A_{k}$, we get that

$$
\left\|v_{k, 2}\right\|_{2,2} \leq\left\|v_{k, 2}\right\|_{\infty, \infty}\left\|v_{k, 2}\right\|_{1,1}=O\left(\frac{\sqrt{\left\|\delta_{k} u\right\|_{2,2}}}{\sigma_{A_{k}}} \frac{\sigma_{A_{k}}}{\left\|\delta_{k} u\right\|_{2,2}^{1 / 4}}\right)=O\left(\left\|\delta_{k} u\right\|_{2,2}^{1 / 4}\right) \rightarrow 0 \quad \text { as } k \uparrow \infty,
$$

which concludes the proof.

In the following corollary we prove an interesting consequence of the previous result.

Corollary 4.6. Suppose that (H1)-(H2) hold true and let $s \in[1, \infty[$. Then, $\bar{u}$ is a strong local solution of $\left(C P^{\prime}\right)$ satisfying the quadratic growth condition iff $\bar{u}$ is a $L^{s}$-weak local solution of $\left(C P^{\prime}\right)$ satisfying the quadratic growth condition.

Proof. If $\bar{u}$ is a $L^{s}$-weak local solution of $\left(C P^{\prime}\right)$ satisfying the quadratic growth condition, then arguing as in the proof of Theorem 4.3 we have that (ii.1) and (ii.2) in Theorem 4.5 are satisfied. Therefore, $\bar{u}$ is a strong local solution of $\left(C P^{\prime}\right)$ satisfying the quadratic growth condition. The other implication being clear (see Remark 2.5), the conclusion follows. 


\section{The case of a strictly convex quadratic Hamiltonian and contin- uous data}

We prove in this section that under a continuity assumption on the data and a convexity condition on the Hamiltonian, the notions of weak and strong minima satisfying the quadratic growth condition are equivalent. We assume that

(H4) (i) We suppose that $a, b \in C(\bar{Q})$ and that there exists $\underline{\ell}>0$ and continuous functions $\ell_{1}: \bar{Q} \rightarrow \mathbb{R}, \ell_{2}: \bar{Q} \times \mathbb{R} \rightarrow \mathbb{R}, \varphi_{1}: \bar{Q} \rightarrow \mathbb{R}$ and $\varphi_{2}: \bar{Q} \times \mathbb{R} \rightarrow \mathbb{R}$ such that

$$
\ell(t, x, y, u)=\ell_{1}(t, x) u^{2}+\ell_{2}(t, x, y), \quad \varphi(t, x, y, u)=\varphi_{1}(t, x) u+\varphi_{2}(t, x, y) \text { and } \ell_{1}(t, x) \geq \underline{\ell} \text { in } \bar{Q} .
$$

(ii) For $\psi=\Phi, \Phi_{E}^{i}, \Phi_{I}^{j}\left(i=1, \ldots, n_{E}\right.$ and $\left.j=1, \ldots, n_{I}\right)$ we assume that $\psi_{y}$ admits a locally Hölder continuous extension to $\bar{\Omega} \times \mathbb{R}$ satisfying $\psi_{y}(x, 0)=0$ for all $x \in \partial \Omega$.

Remark 5.1. (i) $B y(\mathbf{H} 4)$ (i) we have that $\Lambda_{L}(u)=\Lambda_{P}(u)$ for every $u \in \mathcal{K}$.

(ii) By (H4)(ii) and [18, Proposition 2.1], we have the existence of $\left.\beta^{\prime} \in\right] 0,1[$ such that for all $\lambda \in \mathbb{R}^{n_{E}+n_{I}}$ the adjoint state $\bar{p}_{\lambda}$ defined in (3.2) belongs to $C^{\beta^{\prime}, \beta^{\prime} / 2}(\bar{Q}) \cap L^{2}\left([0, T] ; H_{0}^{1}(\Omega)\right)$.

Now we prove the main result of this section, which provides the equivalence of the notions of weak and strong solutions satisfying the quadratic growth condition.

Theorem 5.2. Consider problem $(C P)$ and suppose that $(\mathbf{H 1}),(\mathbf{H 2})$ and $(\mathbf{H} 4)$ are satisfied. Then, for $\bar{u} \in \mathcal{K}$ we have the equivalence of (2.12), (2.14) (for any $s \in[1, \infty[$ ) and (2.16), provided that one of the following conditions is satisfied

(i) The condition (H3) holds true.

(ii) The Robinson constraint qualification (RCQ) holds true and every $v \in C_{\mathcal{K}}(\bar{u})$ satisfies that $v=0$ a.e. in $A_{a}(\bar{u}) \cup A_{b}(\bar{u})$.

Proof. By Remark 2.5 it suffices to prove that (2.12) and (2.16) are equivalent if (i) or (ii) are satisfied. Let us suppose that (i) holds true. We only need to prove, the other implication being trivial, that if $\bar{u}$ satisifies (2.12) then it also satisfies (2.16). Note first that (4.6) implies that $\Lambda_{L}(\bar{u})=\Lambda_{P}(\bar{u})$ is a singleton $\{\lambda\}$ (see [26, Lemma 5.4]). Assumption (H4) implies that $Q_{2}[\bar{u}, \lambda]$ is a Legendre form and so by [26, Theorem 5.7] we obtain that assumptions (i) and (iii) in Theorem 4.4 are satisfied. Thus, in order to conclude we only need to show that the quadratic growth condition (4.7) for the Hamiltonian holds at $(\bar{u}, \lambda)$. Since $\bar{u}$ is a weak solution of the problem $\inf \left\{J(u)-\frac{\alpha}{2}\|u-\bar{u}\|_{2,2}^{2} ; u \in \mathcal{K}\right\}$, by the local Pontryagin principle for weak solution (see [14]), we have the existence of $\hat{\lambda} \in \mathbb{R}^{n_{E}+n_{I}}$ and $\varepsilon>0$ such that

$$
\bar{H}[\hat{\lambda}](t, x)+\frac{\alpha}{2}|u-\bar{u}(t, x)|^{2} \leq H\left(t, x, \bar{y}(t, x), \bar{p}_{\hat{\lambda}}(t, x), u\right) \quad \forall u \in[a(t, x), b(t, x)], \quad|u-\bar{u}(t, x)| \leq \varepsilon,
$$

from which $\hat{\lambda}=\lambda$. Now, for all $(t, x) \in \bar{Q}$ let us define

$$
\hat{u}(t, x)=\underset{u \in[a(t, x), b(t, x)]}{\operatorname{argmin}} H\left(t, x, \bar{y}(t, x), \bar{p}_{\lambda}(t, x), u\right) .
$$

By the strict convexity of the Hamiltonian w.r.t. $u$ and (5.1) we get that $\hat{u}$ is well defined and $\hat{u}=\bar{u}$ a.e. in $Q$. Moreover, using the continuity of the data, assumed in (H4) and the continuity of $\bar{y}$ and $\bar{p}_{\lambda}$, we easily check with (5.2) that $\hat{u}$ is continuous. Therefore, for all $(t, x) \in \bar{Q}, v \in[a(t, x), b(t, x)]$, and $|v-\hat{u}(t, x)| \leq \varepsilon$ we have

$$
H\left(t, x, \bar{y}(t, x), \bar{p}_{\lambda}(t, x), \hat{u}(t, x)\right)+\frac{\alpha}{2}|v-\hat{u}(t, x)|^{2} \leq H\left(t, x, \bar{y}(t, x), \bar{p}_{\lambda}(t, x), v\right) .
$$


Therefore, letting

$$
\beta:=\inf \{H(t, x, \bar{y}(t, x), \bar{p}(t, x), u)-H(t, x, \bar{y}(t, x), \bar{p}(t, x), \hat{u}(t, x)) ;(t, x) \in \bar{Q}, u \in[a(t, x), b(t, x)],|u-\hat{u}(t, x)| \geq \varepsilon\},
$$

the continuity of $\hat{u}$ implies that $\beta>0$. Defining $\alpha^{\prime}=\min \left\{\beta / 4 M^{2}, \frac{\alpha}{2}\right\}$ we obtain that (5.3) holds with $\alpha$ replaced by $\alpha^{\prime}$ and for all $v \in[a(t, x), b(t, x)]$ and so (4.15) is satisfied, which implies the result if (i) holds true. If (ii) holds true, the proof follows the same lines, the only change being the use of [26, Theorem 5.2] instead of [26, Theorem 5.7].

Remark 5.3. Condition (ii) in the above results means that the critical cone is equal to the strict critical cone (see [26]). It is easy to check that this condition is equivalent to an a.e. strict complementarity assumption over the finite dimensional problem appearing in Pontryagin minimum principle.

For problem $\left(C P^{\prime}\right)$ the qualification condition is not needed. The proof of the following result is identical to the previous one.

Theorem 5.4. Consider problem $\left(C P^{\prime}\right)$ and suppose that $(\mathbf{H 1}),(\mathbf{H 2})$ and $\mathbf{( H 4 )}$ are satisfied (where we consider only $\psi=\Phi$ in (H4)(ii)). Then, for $\bar{u} \in \mathcal{K}_{1}$ we have the equivalence of (2.12), (2.14) (for any $s \in\left[1, \infty\left[\right.\right.$ ) and $(2.16)$ (with $\mathcal{K}$ replaced by $\mathcal{K}_{1}$ ).

We end this article with the following example.

Example 5.5. Consider the optimal control problem

$$
\begin{gathered}
\text { inf } \int_{Q}\left[\frac{N}{2} u(t, x)^{2}+\ell(t, x, y(t, x))\right] \mathrm{d} t \mathrm{~d} x, \\
\partial_{t} y-\Delta y+\varphi(y)=u+f \quad \text { in } Q, \\
\text { s.t. } \quad y=0 \quad \text { in } \Sigma, \quad y(0, \cdot)=y_{0}(\cdot), \\
a \leq u \leq b \quad \text { in } Q,
\end{gathered}
$$

where $\ell$ is continuous on $\bar{Q} \times \mathbb{R}$ and satisfies (H2), $\varphi$ is $C^{1}$ and it is increasing, $f \in L^{\infty, \infty}(Q)$, $y_{0} \in W_{0}^{2-\frac{2}{s}, s}(\Omega)$ and $a, b$ are continuous on $\bar{Q}$. Then, for this problem the notions of weak, $L^{s}$-weak (for any $s \in[1, \infty[$ ) and strong solutions satisfying the quadratic growth condition are equivalent.

On the other hand, if we consider the problem with non-convex Hamiltonian

$$
\begin{gathered}
\inf \int_{Q}\left[u(t, x)^{2}-u(t, x)^{4}-y(t, x)^{4}\right] \mathrm{d} t \mathrm{~d} x, \\
\partial_{t} y-\Delta y=u \quad \text { in } Q \\
\text { s.t. } \quad y=0 \quad \text { in } \Sigma, \quad y(0, \cdot)=0 \\
0 \leq u \leq 2 \quad \text { in } Q
\end{gathered}
$$

using [26, Theorem 5.8] we obtain that $\bar{u} \equiv 0$ is a weak local solution satisfying the quadratic growth condition. However, due to the $-u^{4}$ term, we have that $\bar{u}$ is not a $L^{s}$-weak solution for any $s \in[1, \infty[$.

\section{Appendix: proofs of some technical results}

We provide in this section the proofs of Lemma 3.1 and Proposition 3.2 stated in Section 2.

Proof of Lemma 3.1. We have that $\delta y$ satisfies

$$
\begin{aligned}
\partial_{t} \delta y-\Delta \delta y+\left[\int_{0}^{1} \varphi_{y}(t, x, \bar{y}+\tau \delta y, \bar{u}+\tau v) \mathrm{d} \tau\right] \delta y & =-\left[\int_{0}^{1} \varphi_{u}(t, x, \bar{y}+\tau \delta y, \bar{u}+\tau v) \mathrm{d} \tau\right] v \quad \text { in } Q, \\
\delta y & =0 \text { in } \Sigma, \\
\delta y(0, \cdot) & =0 \text { in } \Omega .
\end{aligned}
$$


Using the above equation and equation (3.3) for $z_{1}$, the estimates for $\|\delta y\|_{\mathcal{V}}$ and $\left\|z_{1}[v]\right\| \mathcal{V}$ follow by (H1)(iii) and (2.5). Noting that, omitting the dependence on $(t, x)$,

$$
\begin{aligned}
\varphi(t, x, y[\bar{u}+v], \bar{u}+v)-\varphi(\bar{y}, \bar{u}+v)-\bar{\varphi}(t, x) \delta y & =\int_{0}^{1}[\varphi(t, x, \bar{y}+\tau \delta y, \bar{u}+v)-\bar{\varphi}(t, x)] \mathrm{d} \tau \delta y \\
& =O\left(|\delta y|^{2}+|v||\delta y|\right),
\end{aligned}
$$

we easily check that $d_{1}$ satisfies

$$
\begin{aligned}
\partial_{t} d_{1}-\Delta d_{1}+\bar{\varphi}_{y}(t, x) d_{1} & =O\left(|\delta y|^{2}+|v||\delta y|\right) \text { in } Q, \\
d_{1} & =0 \text { in } \Sigma, \\
d_{1}(0, \cdot) & =0 \text { in } \Omega .
\end{aligned}
$$

In particular, (2.5) implies

$$
\left\|d_{1}[v]\right\|_{2,2}+\left\|d_{1}[v](T, \cdot)\right\|_{2}=O\left(\left[\int_{Q}\left[|\delta y|^{2}+|v \| \delta y|\right]^{2} \mathrm{~d} t \mathrm{~d} x\right]^{\frac{1}{2}}\right)=O\left(\|\delta y\|_{\infty, \infty}\|v\|_{2,2}\right),
$$

which gives the second estimate in (3.5). On the other hand, omitting the dependence on $(t, x)$ and setting $y=y[\bar{u}+v], u=\bar{u}+v$, we have that

$$
\begin{aligned}
\varphi(y, u)-\varphi(\bar{y}, u)-\bar{\varphi}_{y}(t, x) z_{1}-\bar{\varphi}_{y}(t, x) z_{2}-\frac{1}{2} \bar{\varphi}_{y y}(t, x) z_{1}^{2}-\delta \bar{\varphi}_{y}(t, x) z_{1} \\
=\bar{\varphi}_{y}(t, x) d_{2}+\varphi(y, u)-\varphi(\bar{y}, u)-\bar{\varphi}_{y}(t, x) \delta y-\frac{1}{2} \bar{\varphi}_{y y}(t, x) z_{1}^{2}-\delta \bar{\varphi}_{y}(t, x) z_{1} \\
=\bar{\varphi}_{y}(t, x) d_{2}+\varphi_{y}(\bar{y}, u) \delta y+\frac{1}{2} \varphi_{y y}(\bar{y}, u) \delta y^{2}+\int_{0}^{1}(1-\tau)\left[\varphi_{y y}(\bar{y}+\tau \delta y, u)-\bar{\varphi}_{y y}(\bar{y}, u)\right] \mathrm{d} \tau(\delta y)^{2} \\
\quad-\bar{\varphi}_{y}(t, x) \delta y-\frac{1}{2} \bar{\varphi}_{y y}(t, x) z_{1}^{2}-\delta \bar{\varphi}_{y}(t, x) z_{1} \\
=\bar{\varphi}_{y}(t, x) d_{2}+\delta \bar{\varphi}_{y}(t, x) d_{1}+\frac{1}{2} \delta \bar{\varphi}_{y y}(t, x) \delta y^{2}+\frac{1}{2} \bar{\varphi}_{y y}(t, x)\left(\delta y^{2}-z_{1}^{2}\right) \\
\quad+\int_{0}^{1}(1-\tau)\left[\varphi_{y y}(\bar{y}+\tau \delta y, u)-\bar{\varphi}_{y y}(\bar{y}, u)\right] \mathrm{d} \tau(\delta y)^{2} \\
=\bar{\varphi}_{y}(t, x) d_{2}+O\left(\left|d_{1}\right||v|+\left|\delta y^{2}\right||v|+\left|d_{1}\right|\left(|\delta y|+\left|z_{1}\right|\right)+|\delta y|^{3}\right) .
\end{aligned}
$$

This implies that $d_{2}$ solves

$$
\begin{aligned}
\partial_{t} d_{2}-\Delta d_{2}+\bar{\varphi}_{y}(t, x) d_{2} & =O\left(\left|d_{1}\right||v|+\left|\delta y^{2}\right||v|+\left|d_{1}\right|\left(|\delta y|+\left|z_{1}\right|\right)+|\delta y|^{3}\right) \text { in } Q, \\
d_{2} & =0 \text { in } \Sigma \\
d_{2}(0, \cdot) & =0 \text { in } \Omega .
\end{aligned}
$$

Using (2.6) we get that

$$
\left\|d_{2}[v]\right\|_{1,1}+\left\|d_{2}[v](T, \cdot)\right\|_{1}=O\left(\int_{Q}\left[\left|d_{1}\right||v|+\left|\delta y^{2}\right||v|+\left|d_{1}\right|\left(|\delta y|+\left|z_{1}\right|\right)+|\delta y|^{3}\right] \mathrm{d} t \mathrm{~d} x\right),
$$

and the third estimate in (3.5) follows from the previous ones.

Proof of Proposition 3.2. Let us prove (i). Omitting the dependence on $(t, x)$, using the notations introduced in the statement of Lemma 3.1, with $v=\delta u$, and writing $\delta y_{T}(x):=\delta y(T, x)$, we have that

$$
\begin{aligned}
\int_{Q}[\ell(y, u)-\bar{\ell}] \mathrm{d} t \mathrm{~d} x+\int_{\Omega}[\Phi[\lambda](y(T, x))-\bar{\Phi}[\lambda]] \mathrm{d} x= & \int_{Q}[\ell(y, u)-\ell(\bar{y}, u)+\delta \bar{\ell}] \mathrm{d} t \mathrm{~d} x+\int_{\Omega} \bar{\Phi}_{y}[\lambda] \delta y_{T} \mathrm{~d} x \\
& +O\left(\int_{\Omega}\left|\delta y_{T}\right|^{2} \mathrm{~d} x\right), \\
= & \int_{Q}\left[\ell(y, u)-\ell(\bar{y}, u)+\delta \bar{\ell}+\partial_{t} \bar{p}_{\lambda} \delta y+\bar{p}_{\lambda} \partial_{t} \delta y\right] \mathrm{d} t \mathrm{~d} x \\
& +O\left(\int_{\Omega}\left|\delta y_{T}\right|^{2} \mathrm{~d} x\right),
\end{aligned}
$$


where we have integrated by parts in the time variable using that $\bar{p}_{\lambda}(T, \cdot)=\bar{\Phi}[\lambda](\cdot)$ and $\delta y(0, \cdot)=0$. Now, since

$\int_{Q}\left[\ell(y, u)-\ell(\bar{y}, u)-\bar{p}_{\lambda}(\varphi(y, u)-\varphi(\bar{y}, u))\right] \mathrm{d} t \mathrm{~d} x=\int_{Q}\left[\bar{\ell}_{y}-\bar{p}_{\lambda} \bar{\varphi}_{y}\right] \delta y \mathrm{~d} t \mathrm{~d} x+O\left(\int_{Q}\left[|\delta y|^{2}+|\delta y||\delta u|\right] \mathrm{d} t \mathrm{~d} x\right)$,

(where we use that $\bar{p}_{\lambda} \in L^{\infty, \infty}(Q)$, uniformly for $\lambda$ in a bounded set, by the maximum principle), equation (3.2) and the equation satisfied by $\delta y$ imply that

$$
\mathcal{L}(u, \lambda)-\mathcal{L}(\bar{u}, \lambda)=\int_{Q} \delta \bar{H}(t, x) \mathrm{d} t \mathrm{~d} x+O\left(\int_{Q}\left[|\delta y|^{2}+|\delta y||\delta u|\right] \mathrm{d} t \mathrm{~d} x+\int_{\Omega}\left|\delta y_{T}\right|^{2} \mathrm{~d} x\right),
$$

and the result follows from Lemma 3.1. In order to prove (ii), we follow a similar strategy. Expanding up to the second order, we have

$$
\begin{aligned}
\int_{Q}[\ell(y, u)-\bar{\ell}] \mathrm{d} t \mathrm{~d} x= & \int_{Q}[\ell(y, u)-\ell(\bar{y}, u)+\delta \bar{\ell}] \mathrm{d} t \mathrm{~d} x \\
= & \int_{Q}\left[\ell_{y}(\bar{y}, u) \delta y+\frac{1}{2} \ell_{y y}(\bar{y}, u)(\delta y)^{2}+\delta \bar{\ell}\right] \mathrm{d} t \mathrm{~d} x \\
& +\int_{Q} \int_{0}^{1}(1-\tau)\left[\ell_{y y}(\bar{y}+\tau \delta y, u)-\ell_{y y}(\bar{y}, u)\right](\delta y)^{2} \mathrm{~d} \tau \mathrm{d} t \mathrm{~d} x \\
= & \int_{Q}\left[\delta \bar{\ell}_{y} \delta y+\bar{\ell}_{y} \delta y+\frac{1}{2} \bar{\ell}_{y y} z_{1}^{2}+\delta \bar{\ell}\right] \mathrm{d} t \mathrm{~d} x \\
& +O\left(\int_{Q}\left[\left|d_{1}\right|\left(|\delta y|+\left|z_{1}\right|\right)+|\delta u||\delta y|^{2}+|\delta y|^{3}\right] \mathrm{d} t \mathrm{~d} x\right) \\
= & \int_{Q}\left[\delta \bar{\ell}_{y} z_{1}+\bar{\ell}_{y}\left(z_{1}+z_{2}\right)+\frac{1}{2} \bar{\ell}_{y y} z_{1}^{2}+\delta \bar{\ell}\right] \mathrm{d} t \mathrm{~d} x \\
& +O\left(\int_{Q}\left[\left|d_{1}\right||\delta u|+\left|d_{2}\right|+\left|d_{1}\right|\left(|\delta y|+\left|z_{1}\right|\right)+|\delta u \| \delta y|^{2}+|\delta y|^{3}\right] \mathrm{d} t \mathrm{~d} x\right) .
\end{aligned}
$$

Analogously, omitting the $x$ argument and setting $\left(z_{1}\right)_{T}=z_{1}(T, \cdot)$ with a similar convention for $\left(d_{1}\right)_{T}$ and $\left(d_{2}\right)_{T}$,

$$
\begin{aligned}
\int_{\Omega}\left(\Phi[\lambda]\left(y_{T}\right)-\bar{\Phi}[\lambda]\right) \mathrm{d} x= & \int_{\Omega}\left[\bar{\Phi}_{y}[\lambda] \delta y_{T}+\frac{1}{2} \bar{\Phi}_{y y}[\lambda] \delta y_{T}^{2}\right] \mathrm{d} x+O\left(\int_{\Omega}\left|\delta y_{T}\right|^{3} \mathrm{~d} x\right) \\
= & \int_{\Omega}\left[\bar{\Phi}_{y}[\lambda]\left(z_{1}+z_{2}\right)_{T}+\frac{1}{2} \bar{\Phi}_{y y}[\lambda]\left(z_{1}\right)_{T}^{2}\right] \mathrm{d} x \\
& +O\left(\int_{\Omega}\left[\left|\left(d_{2}\right)_{T}\right|+\left|\left(d_{1}\right)_{T}\right|\left|\left(z_{1}\right)_{T}\right|+\left|\left(d_{1}\right)_{T}\right|\left|\delta y_{T}\right|+\left|\delta y_{T}\right|^{3}\right] \mathrm{d} x\right) .
\end{aligned}
$$

Therefore, by Lemma 3.1 and the Cauchy-Schwarz inequality, we get

$$
\begin{aligned}
\mathcal{L}(u, \lambda)-\mathcal{L}(\bar{u}, \lambda)= & \int_{Q}\left[\delta \bar{\ell}_{y} z_{1}+\bar{\ell}_{y}\left(z_{1}+z_{2}\right)+\frac{1}{2} \bar{\ell}_{y y} z_{1}^{2}+\delta \bar{\ell}\right] \mathrm{d} t \mathrm{~d} x \\
& +\int_{\Omega}\left[\bar{\Phi}_{y}[\lambda]\left(z_{1}+z_{2}\right)_{T}+\frac{1}{2} \bar{\Phi}_{y y}[\lambda]\left(z_{1}\right)_{T}^{2}\right] \mathrm{d} x+O\left(\|\delta y\|_{\infty, \infty}\|\delta u\|_{2,2}^{2}\right) .
\end{aligned}
$$

Finally, using (3.3), (3.4), (3.3) and integrating by parts we obtain that

$$
\int_{\Omega} \bar{\Phi}_{y}[\lambda]\left(z_{1}+z_{2}\right)_{T} \mathrm{~d} x=-\int_{Q}\left[\delta \bar{\varphi} \bar{p}+\delta \bar{\varphi}_{y} z_{1} \bar{p}+\frac{1}{2} \bar{\varphi}_{y y} z_{1}^{2} \bar{p}+\bar{\ell}_{y}\left(z_{1}+z_{2}\right)\right] \mathrm{d} t \mathrm{~d} x,
$$

which combined with (5.4) yields (3.8).

Acknowledgment: The authors express their gratitude to the authors of $[8,9]$ for valuable discussions.

\section{References}

[1] J. P. Aubin. Un théorème de compacité. C. R. Acad. Sci. Paris, 256:5042-5044, 1963.

[2] H. T. Banks and K. Kunisch. Estimation techniques for distributed parameter systems. Systems \& Control: Foundations \& Applications. Birkhäuser Boston, Inc., Boston, MA, 1989. 
[3] T. Bayen, J.F. Bonnans, and F. J. Silva. Characterization of local quadratic growth for strong minima in the optimal control of semi-linear elliptic equations. Trans. Amer. Math. Soc., 366:2063-2087, 2014.

[4] T. Bayen and F.J. Silva. Weak and strong minima : from calculus of variation towards pde optimization. IFAC Workshop on Control of Systems Modeled by Partial Differential Equations, 2013.

[5] M. Bergounioux and N. Merabet. Sensitivity analysis for optimal control of problems governed by semilinear parabolic equations. Control Cybernet., 29(4):861-886, 2000.

[6] O.V. Besov, V.P. Il'in, and S.M. Nikol'skii. Integral representations of functions and imbedding theorems. "Nauka", Moscow, 1979.

[7] J. F. Bonnans and E. Casas. Contrôle de systèmes elliptiques semilinéaires comportant des contraintes sur l'état. In Nonlinear partial differential equations and their applications. Collège de France seminar, Vol. VIII (Paris, 1984-1985), volume 166 of Pitman Res. Notes Math. Ser., pages 69-86. Longman Sci. Tech., Harlow, 1988.

[8] J. F. Bonnans, X. Dupuis, and L. Pfeiffer. Second-order necessary conditions in pontryagin form for optimal control problems. SIAM J. Control Optim. to appear, 2014.

[9] J. F. Bonnans, X. Dupuis, and L. Pfeiffer. Second-order sufficient conditions for strong solutions to optimal control problems. ESAIM-COCV, 159(1):1-40, 2014.

[10] J.F. Bonnans and P. Jaisson. Optimal control of a time-dependent state constrained parabolic equation. SIAM J. Control Optim., 48-7:4550-4571, 2010.

[11] J.F. Bonnans and H. Zidani. Optimal control problems with partially polyhedric constraints. SIAM J. Control Optim., 37(6):1726-1741 (electronic), 1999.

[12] E. Casas. Pontryagin's principle for state-constrained boundary control problems of semilinear parabolic equations. SIAM J. Control Optim., 35-4:1297-1237, 1997.

[13] E. Casas and M. Mateos. Second order optimality conditions for semilinear elliptic control problems with finitely many state constraints. SIAM J. Control Optim., 40(5):1431-1454 (electronic), 2002 .

[14] E. Casas, J.-P. Raymond, and H. Zidani. Pontryagin principle for local solutions of control problems with mixed state control constraints. SIAM J. Control Optim., 39-4:1182-1203, 2000.

[15] E. Casas and F. Tröltzsch. Second order necessary optimality conditions for some stateconstrained control problems of semilinear elliptic equations. Appl. Math. Optim., 39:211-228, 1999.

[16] E. Casas and F. Tröltzsch. Second order necessary and sufficient optimality conditions for optimization problems and applications to control theory. SIAM J. Optim., 13:406-431, 2002.

[17] H. Goldberg and F. Tröltzsch. Second order sufficient optimality conditions for a class of nonlinear parabolic boundary control problems. SIAM J. Control Optim., 31:1007-1025, 1993.

[18] B. Hu and J. Yong. Pontryagin maximum principle for semilinear and quasilinear parabolic equations with pointwise state constraints. SIAM J. Control Optim., 33-6:1857-1880, 1995. 
[19] J.-P. Kernevez. Enzyme mathematics. Studies in Mathematics and its Applications. NorthHolland Publishing Co., Amsterdam-New York, 1980.

[20] O. A. Ladyzhenskaya, V.A. Solonnikov, and N.N. Ural'ceva. Linear and quasilinear equations of parabolic type. Translation of Mathematical Monographs, Vol. 23. American Mathematical Society, Providence, R.I., 1967.

[21] J.-L. Lions and E. Magenes. Problèmes aux limites non homogènes et applications. Vol. 1. Travaux et Recherches Mathématiques, No. 17. Dunod, Paris, 1968.

[22] A. Milyutin and N. Osmolovskiǔ. Calculus of Variations and Optimal Control. AMS., 1998. Systems and Control: Foundations and Applications.

[23] P. Neittaanmaki and D. Tiba. Optimal Control of Nonlinear Parabolic Systems: Theory, Algorithms and Applications. Chapman \& Hall/CRC Pure and Applied Mathematics. Taylor \& Francis, 1994.

[24] S. M. Robinson. Strongly regular generalized equations. Mathematics of Operations Research, $5(1): 43-62,1980$.

[25] A. Shapiro. Perturbation analysis of optimization problems in Banach spaces. Numer. Funct. Anal. Optim., 13(1-2):97-116, 1992.

[26] F.J. Silva. Second order analysis for the optimal control of parabolic equations under control and final state constraints. Preprint, 2014.

[27] J. Simon. Compact sets in $L^{p}(0, T ; B)$. Annali Mat. Pura appl. (IV), CXLVI:65-96, 1987.

[28] F. Tröltzsch. Optimal Control of Partial Differential Equations - Theory, Methods and Applications. Graduate Studies in Mathematics, Vol. 112. American Mathematical Society, Providence, Rhode Island, 2010.

[29] G. F. Webb. Theory of nonlinear age-dependent population dynamics. Monographs and Textbooks in Pure and Applied Mathematics. Marcel Dekker, Inc., New York, 1985. 